\title{
Sensitivity of Basin-Wide Meridional Overturning to Diapycnal Diffusion and Remote Wind Forcing in an Idealized Atlantic-Southern Ocean Geometry
}

\author{
Barry A. Klinger ${ }^{1}$, Sybren Drijfhout ${ }^{2}$, Jochem Marotzke ${ }^{3}$, and Jeffery R. Scott ${ }^{4}$
}

August, 2001

Submitted to J. Phys. Oceanogr.

\footnotetext{
${ }^{1}$ Nova Southeastern University Oceanographic Center, Dania Beach, FL 33004. Currently at George Mason University, Fairfax, VA 22030 and Center for Ocean-Land-Atmosphere Studies, Calverton, MD, 20705; klinger@cola.iges.org.

Address: Royal Netherlands Meteorological Institute (KNMI), PO Box 201, 3730 AE De Bilt, The Netherlands.

${ }^{3}$ School of Ocean and Earth Science, University of Southampton, Southampton S014 3ZH, United Kingdom.

${ }^{4}$ MIT, Cambridge, MA 02139 USA
} 


\begin{abstract}
Recent numerical experiments indicate that the rate of meridional overturning associated with North Atlantic Deep Water is controlled both by mixing and by windstress in the Southern Ocean, where the zonal periodicity of the domain alters the nature of the flow. We find a simple approximate expression for meridional overturning as a solution to Gnanadesikan's (1999) cubic scale relation. We compare the predicted overturning to coarse-resolution numerical experiments with an idealized Atlantic-Ocean/Southern Ocean geometry. The scaling accurately predicts the sensitivity to forcing for experiments with a level model employing isopycnal diffusion of temperature, salinity, and "layer thickness". A layer model produces similar results, increasing our confidence in the numerics of both models. Level model experiments with horizontal diffusivity have similar qualitative behavior but somewhat different sensitivity to forcing.
\end{abstract}

We highlight the difference in meridional overturning induced by changes in windstress or vertical diffusivity. Wind-driven circulation anomalies outside the region of windstress perturbation include strongly cross-isopycnal flow near the surface and approximately along-isotherm flow in the thermocline. Overturning anomalies far from the windstress perturbations are not completely determined by windstress in the zonally-periodic Southern Ocean: windstress outside the periodic region strongly influences the transport of heat across the equator primarily by changing the temperature of the flow across the equator. Most of the total meridional heat transport across the basin can be decomposed into contributions due to the westerlies, easterlies, and vertical diffusivity; here we show how the westerlies contribution is related to the surface temperature profile. 


\section{Introduction}

Toggweiler and Samuels $(1995,1998)$ introduced the radical idea that a substantial part of the "thermohaline circulation" may in fact be driven by windstress. Since there can not be a net meridional geostrophic flow at any latitude and depth at which there are no zonal boundaries, they argue that the northward Ekman transport in the Southern Ocean must return below the sill depth of the Drake Passage, about $2500 \mathrm{~m}$. The northward surface Ekman transport then connects to the southward deep geostrophic flow via a sinking in the northern North Atlantic, where the stratification is weak enough to allow significant deep water formation. This North Atlantic Deep Water (NADW) cell is thus driven by both the classical interior diapycnal mixing (see for instance Bryan, 1987) and by the strength of the southern wind.

Toggweiler and Samuels' studies (as well as a further examination by McDermott, 1996) used global ocean models with coarse resolution and with eddy mixing parameterized by horizontal diffusion. However, it may be more appropriate to parameterize eddies with along-isopycnal mixing and with the diffusion of "thickness" (vertical distance between neighboring isopycnals) representing the release of available potential energy by baroclinic instability (Gent and McWilliams, 1990; Visbeck et al. 1997). Eddy fluxes of mass and buoyancy are likely to be especially important in the Southern Ocean, for just the reason that fluxes by direct meridional currents are inhibited. Therefore it is interesting to examine whether the sensitivity of NADW overturning strength to forcing depends on the eddy parameterization. While other studies have examined differences between general circulation models using the different eddy parameterizations (Danabasoglu et al., 1994; Danabasoglu and McWilliams, 1995; Hirst and McDougall, 1998), these did not explicitly look at NADW cell sensitivity to different forcing. 
Recently, Gnanadesikan (1999, henceforth G99) proposed a scaling for the strength of the NADW cell as a function of vertical diffusivity $\kappa_{V}$, southern ocean windstress $\tau_{S}$, and eddy thickness diffusivity, $\kappa_{H}$. We extend this work in several directions. The G99 scaling consists of a cubic equation whose solution is not apparent by inspection. Since part of the appeal of scaling rules is to provide an easily understandable grasp of quantitative relationships, it would be useful to find an explicit formula relating overturning properties to forcing and other external parameters. After nondimensionalizing the system, we show that the cubic has a simple approximate solution which can be used to obtain better intuition about the physical system (Section 2 below).

G99 tested the scaling with a series of global, coarse resolution numerical experiments with isopycnal mixing and thickness diffusion. We do a direct comparison of otherwise identical runs using the two common mixing schemes, horizontal $(\mathrm{H})$ and isopycnal/GentMcWilliams (GM). These numerical experiments use the GFDL Modular Ocean Model, which has fixed vertical levels. We also test these results with an isopycnal layer model (I), which allows for a numerically cleaner representation of isopycnal mixing. Our configuration is simpler than the global configuration of G99 and others, consisting of a single basin in which the zonal boundaries are replaced by periodic boundary conditions in the latitude range of the Southern Ocean, a region we refer to as the "Channel."

Toggweiler and Samuels' explanation of how windstress over the Southern Ocean influences the NADW cell focused on the influence of the periodic channel, which is in the vicinity of $55^{\circ} \mathrm{S}$. This picture was based on numerical experiments which compared runs with wind altered in the wider latitude range south of $30 \mathrm{~S}$ (as was the case for McDermott, 1996, and Gnanadesikan, 1999). In contrast, the numerical experiments of Tsujino and Suginohara (1999) showed that adding wind to one hemisphere enhanced the deep thermohaline cell across much of the domain in a basin with no periodic channel at all. Tsujino and Sugi- 
nohara's results raise the question of whether it is not merely the wind in the Drake Passage latitudes that affects the NADW cell, but that wind equatorward of the Passage can have an effect. More fundamentally, the closed-basin experiments raise the question of whether we understand what determines how much of subpolar meridional Ekman transport can be returned "locally" (within the subpolar region near the forcing) and how much must make a basin-wide circuit. We conduct further sensitivity experiments to explore how much of the Drake Passage Effect is really due to wind in the Drake Passage.

The strengthening of the NADW cell by the wind in the Southern Ocean introduces a fundamental asymmetry between the northern and southern heat transport, with the ocean driving northward heat transport in both hemispheres. Since the heat transport is a key variable in ocean-atmosphere interaction, it is useful to be able to predict the size of this asymmetric component. Our single basin study has several components-including thermohaline overturning, windstress forcing, and the influence of the channel-which contribute to the heat transport. We analyze the heat transport in terms of the individual components, especially the influence of windstress poleward of latitude $30^{\circ}$ on the heat transport.

\section{Scaling for Southern Winds and Uniform Diapycnal Diffusion}

G99 combined the classical "advective-diffusive" scaling (Bryan and Cox, 1967; Bryan, 1987; Colin de Verdiere, 1988) with a wind-driven component by assuming that the volume transport is given by

$$
T_{N}=T_{D}+T_{S}
$$

where $T_{N}$ is the total NADW cell strength, $T_{D}$ is the component associated with diffusion over nearly the entire domain, and $T_{S}$ is the component associated with the southern wind. 
G99's scaling was explicitly based on western boundary current dynamics, but one can arrive at the same scaling using geostrophy, which implies that

$$
T_{N}=c \Delta b D^{2} / f_{0}
$$

where $D$ is the pycnocline depth scale, $f_{0}$ is the typical midlatitude value of the Coriolis parameter, and $\Delta b$ is the pole-to-equator range of surface buoyancy $(=g \Delta \rho / \rho$, for gravitational acceleration $g$, density range $\Delta \rho$ and density $\rho$ ). The meridional transport is directly related to the zonal density difference, but there is reason to believe that the two buoyancy scales are proportional, with a proportionality constant $c$ (Park and Bryan, 2000; Marotzke, 1997). The diffusive component of the cell is given by

$$
T_{D}=\kappa_{V} A / D
$$

where $A$ is the surface area of upwelling (presumably most of the basin). Finally, G99 argued that Southern Ocean contribution was given by the Ekman transport minus a contribution due to eddy fluxes across the tilting isopycnals in the Southern Ocean, or

$$
T_{S}=\left(\frac{\tau}{\rho f_{S}}-\kappa_{I} \frac{D}{L_{y}}\right) L_{x}
$$

where $f_{S}$ is the Coriolis parameter in the Southern Ocean, $\kappa_{I}$ is the thickness diffusivity, $L_{y}$ is the width of the Southern Ocean frontal zone, and $L_{x}$ is the length of the Southern Ocean. The resulting cubic equation in $D$ is

$$
\left(\frac{c \Delta b}{f_{0}}\right) D^{3}+\left(\kappa_{I} \frac{L_{x}}{L_{y}}\right) D^{2}-\left(\frac{\tau L_{x}}{\rho f_{S}}\right) D-\kappa_{V} A=0
$$

The strength of the NADW cell can be calculated from $D$ via (3). It should be noted that using the same scale vertical lengthscale $D$ for horizontal velocity (equation (2)) and density (equation (3)) is problematical (Scott, 2000), but here we will follow G99's assumptions. In the case of no wind and no thickness diffusion $\left(\tau=0\right.$ and $\left.\kappa_{I}=0\right)$, we recover the classical 
advective-diffusive relationships from (5):

$$
D_{0}=\left(\frac{\kappa_{v} A f_{0}}{c \Delta b}\right)^{1 / 3}
$$

and

$$
T_{0}=\left(c \Delta b \kappa_{V}^{2} A^{2} / f_{0}\right)^{1 / 3}
$$

Nondimensionalizing (5) reduces the number of variables and makes it somewhat easier to see patterns in the solution. There are many possible sets of quantities to use as scales, but $D_{0}$ and $T_{0}$ stand out as being especially familiar quantities. Then we can define $d=D / D_{0}$, a measure of how different the pycnocline depth is from its classical value (at $d=1$ ), and (5) becomes

$$
d^{3}+S d^{2}-E d-1=0
$$

Here the wind strength is given by

$$
E=\frac{\tau L_{x} / \rho f_{S}}{T_{0}}=\frac{\text { Ekman Transport }}{\text { Diffusive Transport }}
$$

and the strength of horizontal eddy mixing is given by

$$
S=\frac{L_{x}}{L_{y}} \frac{\kappa_{I} / A}{\kappa_{V} / D_{0}^{2}},
$$

which does not have as straightforward a physical interpretation as $E$. However, we can define a meridional length scale $M$ such that $A=L_{x} M$, in which case,

$$
S=\frac{D_{0}^{2} / \kappa_{V}}{L_{y} M / \kappa_{I}}
$$

This can be interpreted as a ratio of timescales, where the numerator is the diffusive timescale for the thermocline and the denominator is a more abstract timescale associated with horizontal thickness diffusion. Using the above scaling, we can also define a nondimensional cell strength $t_{N}=T_{N} / T_{0}$. From (2), (6) and (7), this becomes

$$
t_{N}=d^{2}
$$


For small $E$ and $S$, a first order perturbation solution to (8) around $d=1$ yields

$$
d \approx 1+(1 / 3)(E-S)
$$

Equation (8) can be solved numerically. We choose the parameter range $0<S<1$ and $0<E<6$. Parameter values too large for this range occur as $\kappa_{V} \rightarrow 0$, in which case the classical scaling does not provide convenient scales for nondimensionalization. As we will see, our numerical experiments stay inside this range even for $\kappa_{V}$ as low as $.05 \mathrm{~cm}^{2} / \mathrm{s}$. Within this range, we find that the contours of $d(E, S)$ are well-approximated by straight lines. Using this fact and doing some empirical curve fitting, we find that the approximation

$$
d=\frac{1+.28 E-.055 S}{1+.22 S}
$$

is within .05 of the exact solution in this parameter range. Note that this is fairly close to the perturbation solution (13).

Table 1 displays the key dimensional and nondimensional parameters discussed above for several values of $\kappa_{V}, \tau$, and basin width corresponding to the numerical experiments described in the next two sections. Low and high wind cases have $\tau$ of .06 and $.18 \mathrm{~N} / \mathrm{m}^{2}$, respectively, corresponding to .5 and 1.5 times observed windstress over the Southern Ocean. The zonally periodic channel is assumed to be at $50^{\circ} \mathrm{S}$. Default and wide basin widths are $60^{\circ}$ and $240^{\circ}$ longitude, respectively. Basin lengths (for calculating $A$ ) are $120^{\circ}$ latitude and $L_{y}=1000 \mathrm{~km}$. Surface buoyancy range is $.05 \mathrm{~m} / \mathrm{s}^{2}$ (similar to the real world), $c=1$, and $\kappa_{I}=1000 \mathrm{~m}^{2} / \mathrm{s}$, a value typical for coarse resolution numerical models and perhaps the right order of magnitude for parameterizing mesoscale eddies in the real world.

For these values, Table 1 shows that $S$ (a measure of the relative importance of lateral thickness diffusion and vertical density diffusion) is small in all cases but not negligible for low $\kappa_{V}$ or for a wide basin. The Southern Ocean Ekman transport can be smaller or larger 
than $T_{0}$ (as measured by $E$ ), for various combinations of $\tau$ and $\kappa$ presented here. We can see from $d$ (or by comparing $T_{N}$ to $T_{0}$ ) that the total overturning is not very sensitive to $\tau$ if $\kappa_{V}=.05 \mathrm{~cm}^{2} / \mathrm{s}$ but is quite sensitive for $\kappa_{V}=.5 \mathrm{~cm}^{2} / \mathrm{s}$. The actual value of $\kappa_{V}$ in the ocean is rather poorly constrained and geographically nonuniform, ranging from $\mathrm{O}\left(.1 \mathrm{~cm}^{2} / \mathrm{s}\right)$ in the thermocline to $\mathrm{O}\left(1.0 \mathrm{~cm}^{2} / \mathrm{s}\right)$ in abyssal water (Gregg, 1987; Ledwell et al., 1993; Toole et al., 1997; Polzin et al., 1997).

What is the relative importance of the classical component due to vertical diffusion and the wind-driven component of the meridional overturning? In view of the observational uncertainties, we pose the question another way. The wind contribution grows in importance as $\tau$ increases, and as $\kappa_{V}$ and $\kappa_{I}$ decrease. Given $\tau$ in the Southern Ocean, how weak would $\kappa_{V}$ have to be in order for the actual overturning to be at least twice as large as $T_{0}$ ? To estimate this, we first invert (14) to find $E(S)$ for $d^{2}=t_{N}=2$. We don't know what $S$ should be, but guided by the results in Table 1, we produce estimates for $S=0$ (for which $E(S)=1.5$ ) and $S=.5$ (for which $E(S)=2.1$ ). In the real world, $\tau \approx .12 \mathrm{~N} / \mathrm{m}^{2}$ over a zonal distance of $360^{\circ}$, which produces an Ekman transport $T_{E}$ of $42 \mathrm{~Sv}$. For calculating $T_{0}$, the relevant area of the world ocean is roughly equal to that of a sector about $240^{\circ}$ wide. Since $E=T_{E} / T_{0}$, we use (7) to find the $\kappa_{V}$ which results in the desired $E$ and $T_{E}$. For $S=0$, the overturning is at least doubled if $\kappa_{V} \leq .23 \mathrm{~cm}^{2} / \mathrm{s}$, while for $S=.5$, we must have $\kappa_{V} \leq .13$

\section{$3 \quad$ Numerical Models}

Two different numerical models are used. One is the Geophysical Fluid Dynamics Laboratory Modular Ocean Model (MOM) version 2.2 (Pacanowski, 1996), a primitive equation model in spherical coordinates in the horizontal and constant-depth coordinates in the vertical. MOM 
is a "B-grid model" (Arakawa and Lamb, 1977), with horizontal velocity grid locations offset by half a gridspacing in latitude and longitude from temperature gridpoints. The second model is an isopycnal primitive equation model. The model is based on the code of Bleck and Boudra (1986).

\subsection{Level Model}

The basin is a sector that ranges from $66^{\circ} \mathrm{S}$ to $62^{\circ} \mathrm{N}$ (measuring from the centers of temperature grid-cells), is $60^{\circ}$ wide, and is $4500 \mathrm{~m}$ deep. There are 16 vertical levels ranging in thickness from approximately $52 \mathrm{~m}$ at the top to approximately $510 \mathrm{~m}$ at the bottom. In most of the experiments, the horizontal gridspacing is $2^{\circ}$ in both horizontal directions; some sensitivity tests have $1^{\circ}$ resolution and others have $4^{\circ}$ in latitude and $3.75^{\circ}$ in longitude. From $64 \mathrm{~S}$ to $48 \mathrm{~S}$ (measuring from the centers of temperature grid-cells), the basin is periodic in longitude, thus representing the topology of the Southern Ocean. We refer to this area as the "Channel". Because the zonal boundaries in MOM are generated by filling grid-cells with "land," the Channel has an extra $2^{\circ}$ of width. In order to represent the ridge at the eastern edge of the Scotia Sea, which in the real ocean is somewhat to the east of the Drake passage, the extra grid-cell has a depth of about $2545 \mathrm{~m}$. The Southern Ocean passage is simpler and wider than the Drake Passage, which has land north of about $55^{\circ} \mathrm{S}$ as well as the Sandwich Islands emerging from the ridge to its east. These approximations are appropriate to the coarse resolution of the model.

Our "twin" experiments have different forms for lateral diffusion of temperature $T$ and salinity $S$. In "H" experiments, $T$ and $S$ are simply diffused horizontally $\left(\kappa_{H}=1000 \times\right.$ $\left.10^{4} \mathrm{~cm} 2 / \mathrm{s}\right)$. In "GM" experiments, $T$ and $S$ are diffused along isopycnals. In addition, the model calculates a "bolus" velocity which is added to the traditional velocity components. This velocity parameterizes correlations between eddy variability in density and in velocity 
(unresolved by the model) and is equivalent to the lateral diffusion of "thickness," $d \rho / d z$, where $z$ is the vertical coordinate (Gent and McWilliams, 1990). In GM runs, lateral diffusion of $T, S$, and thickness are all $\kappa_{I}=1000 \times 10^{4} \mathrm{~cm}^{2} / \mathrm{s}$; the full diffusivity tensor is used rather than a small-angle approximation. Both H and GM runs also have horizontal viscosity $\nu_{H}=250,000 \times 10^{4} \mathrm{~cm}^{2} / \mathrm{s}$ (giving a western boundary current width appropriate to the coarse resolution) and vertical viscosity $\nu_{V}=100 \mathrm{~cm}^{2} / \mathrm{s}$. Different experiments have "high" vertical diffusivity of $\kappa_{V}=.5 \mathrm{~cm}^{2} / \mathrm{s}$ and "low" diffusivity of $\kappa_{V}=.05 \mathrm{~cm}^{2} / \mathrm{s}$. The basin walls and bottom have insulating and no-slip boundary conditions. In all experiments, the advective terms in the model's temperature and salinity evolution equations use the fluxcorrected transport scheme, which is a compromise between the overly-diffusive upstream difference scheme and the property-altering centered-difference scheme (Gerdes et al., 1991).

Surface level $T$ and $S$ are strongly restored to zonally-uniform reference profiles with a fairly restoring timescale of $30 \mathrm{~d}$. The behavior of the circulation can be different when a weaker restoring is used (Rahmstorf and England, 1997). The reference profiles are symmetric about the equator and loosely based on realistic SST and SSS profiles for the ocean:

$$
T_{R}=28(.5[1+\cos (\pi \phi / 68)])^{3 / 4},
$$

where $\phi$ is latitude (in degrees), and

$$
\begin{array}{cc}
S_{R}=34+4.125 e^{-(\phi / 25)^{2}} \sin (\pi \phi / 50)^{2} & (|\phi|<50) \\
S_{R}=34 & (|\phi|>50)
\end{array}
$$

(see Figure 1a). In all experiments, density is calculated via an approximation to the standard nonlinear equation of state. The surface density based on $T_{R}$ and $S_{R}$ is shown in Figure 1b.

FIGURE 1 Surface forcing as a function of latitude for experiments. Restoring profiles for (a) temperature (solid, left axis labels) and salinity (dashed, right axis labels), and (b) density 
as measured by $\sigma_{0}$ (solid) and $\sigma_{2}$ (dashed, offset by $9.3115 \mathrm{~kg} / \mathrm{m}^{3}$ ). (c) Zonal windstress for weak wind (heavy solid), strong southern wind (solid), strong channel wind (solid with dots) and strong northern and southern wind (dashed). (d) Meridional Ekman volume transport; same linestyles as in (c).

The model is also driven by zonally-uniform zonal windstress. The profiles are based on the smoothed, annually and zonally averaged Hellerman and Rosenstein (1983) data. South of $30^{\circ} \mathrm{S}$, the "Weak Wind" case has windstress multiplied by .5, while the "Strong Wind" case has windstress multiplied by 1.5 (Figure 1c,d). Experiments with low diffusivity and each of the two wind strengths were also conducted in a wider basin, $240^{\circ}$ longitude wide. The larger basin surface area and Southern Ocean zonal extent are more like the real world than the narrow-basin experiments, and provide a larger wind- and buoyancy-driven overturning signal with which to test the scaling.

Further wind-sensitivity experiments are conducted with "Northern Winds" and "Channel Winds". For the Northern Winds experiment, wind is identical to the Strong Wind except north of $30 \mathrm{~N}$, which has an anomaly equal to the difference between Strong and Weak winds (Figure 1c,d). In symbols, if we let denote Strong, Weak and Northern winds by $\tau_{S}(\phi), \tau_{W}(\phi), \tau_{N}(\phi)$, respectively, then for $\phi>30^{\circ}$,

$$
\tau_{N}(\phi)=\tau_{S}(\phi)+\left(\tau_{S}(-\phi)-\tau_{N}(-\phi)\right)
$$

The Channel Winds experiment is similar to the Strong Winds experiment but with the strengthened winds confined to the Channel and poleward (Figure 1c,d):

$$
\tau_{C}(\phi)=\tau_{W}(\phi)+\left(\tau_{S}(\phi)-\tau_{W}(\phi)\right) W(\phi)
$$

where $W=1.0$ between the southern boundary and $51 \mathrm{~S}, W=0.0$ between $45 \mathrm{~S}$ and the northern boundary, and $W$ varies linearly between $45 \mathrm{~S}$ and $51 \mathrm{~S}$.

In each experiment, the system is integrated forward in time until the trend in peak meridional overturning is small (typically no more than .01 Sv/century; $1 \mathrm{~Sv} \equiv 10^{6} \mathrm{~m}^{3} / \mathrm{s}$ ) and 
decreasing. Unequal time steps are taken for the momentum (1 hr) and temperature and salinity (typically 5 days) evolution equations, as described by Bryan (1984). The approach to equilibrium is further accelerated by occasionally extrapolating exponentially decaying trends in temperature and salinity (Klinger, 2000). Typical integration times were 1000 to 5000 years as measured by the temperature evolution equation.

\section{$3.2 \quad$ Isopycnal Model}

The domain of the model, bottom topography and resolution are as in the level model, as is the isopycnal and vertical diffusion and horizontal viscosity. The isopycnal model omits vertical viscosity; see Bleck and Boudra (1986). The model also uses a linear equation of state; the twelve isopycnal layers have $\sigma_{0}$ values of, respectively: $21.73,22.73,23.53,24.33$, 24.93, 25.43, 25.93, 26.33, 26.73, 27.03, 27.23, 27.33.

The present model is an extension of the model described in Drijfhout (1994). It contains more vertical resolution and diapycnal diffusion according to Huang and Bryan (1987). Splitting between barotropic and baroclinic mode is according to Bleck and Smith (1990). The model takes unequal time time steps in momentum and density (Bryan, 1984) as with the level model. The wind stress is assumed to decrease linearly within a layer of 50 $\mathrm{m}$, the bottom stress decreases linearly within a layer of $25 \mathrm{~m}$. The treatment of buoyancy forcing follows the methodology described in Drijfhout (1994). Both models are driven by equivalent wind and buoyancy forcing.

The level model relaxes both temperature and salinity to prescribed SST and SSS profiles with a restoring timescale of $t_{R}=30$ days. This is equivalent to restoring density to the profile shown in Figure $1 \mathrm{~b}$ with $t_{R}=30$ days. The layer model directly relaxes density with a restoring timescale of 30 days to the profile shown in Figure 1b. To calculate the 
buoyancy flux through the surface we use the layer weighted density of the upper $50 \mathrm{~m}$ for restoring. This buoyancy flux is used to drive a mass exchange between surface layer (the first layer containing mass) and the layer below. The increase/decrease of the surface layer, $\Delta h$ is given by:

$$
\Delta h=\frac{50 \Delta t\left(\rho_{s}-\rho_{*}\right)}{t_{R} \Delta \rho}
$$

where $\Delta t$ is the model timestep, $\rho_{s}$ is the layer weighted density of the upper $50 \mathrm{~m}, \rho_{*}$ is the reference density for restoring, and $\Delta \rho$ is the density difference between surface layer and layer below.

If $\Delta h$ is negative and larger than the thickness of the surface layer, the remaining part of the buoyancy flux is used for mass exchange between the two uppermost subsurface layers (when present). If $\Delta h$ is positive and there is a massless layer with density larger than the reference density, then the buoyancy flux is used to fill up this massless layer. The mass exchange due to diapycnal diffusion is applied after the mass exchange due to surface buoyancy gain/loss. Momentum is exchanged between layers, consistent with this mass exchange. The runs have been integrated for 1500 years. At the end of the integration the trend in the stratification is small and decreasing at a rate such that the streamfunction values at $1500 \mathrm{yr}$ are likely to be within $1 \%$ of their final values.

\section{Numerical Results}

\subsection{Overturning Strength and Scaling}

There are several definitions of the meridional overturning cells. While the more straightforward definition is to calculate a streamfunction $\Phi_{z}$ based on the zonal integral of meridional velocity $v$ at every depth $z$, this does not highlight the watermass transformation. An alternative streamfunction, $\Phi_{\rho}$ can be computed by taking the zonal integral of $v$ at constant 
density, so that "vertical" flow in the $\rho$ - $\phi$ plane can be interpreted as net cross-isopycnal flow. To take one notable example, $\Phi_{z}$ calculated in global models has a relatively strong Deacon Cell, in which northward Ekman transport in the Drake Passage latitudes sinks to great depths just north of the Drake Passage, returns southward, and upwells just south of the Drake Passage. One might interpret the strong downwelling in the stratified region north of the Drake Passage as indicating strong cooling, but much of the apparent transformation is actually an artifact of the zonal tilt of the isotherms. The $\Phi_{\rho}$ shows little cooling in this region (Doos and Webb, 1994; Hirst et al., 1996). In the GM experiments, one can also look at advective, eddy (refering to the bolus velocity which parameterizes eddy-induced transport that is unresolved by the model) or total (advective plus eddy) components of velocity. For $\Phi_{z}$, the Deacon Cell is strong in the advective component of the overturning but much smaller in the total overturning because the eddy component has a strong cell flowing opposite the Deacon Cell (Hirst and McDougall, 1998). $\Phi_{\rho}$ shows similar compensation between an advective component Deacon Cell and an opposite eddy component cell, but the cells are less than half as strong as their $\Phi_{z}$ counterparts (ibid.). In the discussion below, "overturning" will refer to $\Phi_{\rho}$ (including eddy component for the GM experiments) unless noted otherwise. $\Phi_{\rho}$ was calculated on $\sigma_{2}$ surfaces. In theory one should use neutral surfaces instead (McDougall, 1987; Hirst et al., 1996), but in practice this is only an important issue when there are large $S$ gradients on $T$ surfaces, which is not the case over most of the domain in our experiments. $\Phi_{r} h o$ was also calculated on $\sigma_{0}$ surfaces for the Strong Wind, high $\kappa_{V}$ case, with nearly identical results.

Channel experiments are repeated at several different horizontal resolutions. The Appendix discusses the sensitivity of the model overturning to resolution. Here we refer to $2^{\circ}$ resolution experiments except where otherwise noted. 
All numerical experiments have an NADW cell consisting of sinking near the northern boundary, dense southward flow, and return flow closer to the surface (Figures 2). Note that surface zonal density variations are small (due to the coarse resolution and strong restoring); only streamlines in the lightest water at each latitude represent surface flow. Overturning streamfunctions are qualitatively similar for GM (Figure 2), H (Figure 3), and layer model (Figure 4) experiments. Shallow "Subtropical Cells" (McCreary and Lu, 1994) driven by poleward Ekman transport dominate the lightest water. There is generally some sign of an abyssal counter-rotating "AABW cell" (Antarctic Bottom Water) which is barely visible in the $\Phi_{\rho}$ figures. The NADW cell grows stronger when either $\tau$ or $\kappa_{V}$ are increased. In the Weak Wind cases, the NADW cell does not extend to near the southern boundary; a single southern-sinking cell consisting of a merged AABW cell and Subtropical Cell dominates the southern hemisphere. In the Strong Wind case, the northward Ekman transport south of $30^{\circ} \mathrm{S}$ extends the NADW cell to near the southern boundary.

FIGURE 2 Meridional overturning for low and high vertical diffusivity $\kappa_{V}$ and low and high southern wind $\tau$, GM runs including both advective and eddy components. Contour interval $=1 \mathrm{~Sv}$. Overturning with $\sigma_{2}$ coordinate is calculated using 51 unequally spaced density intervals which give higher resolution for higher $\sigma_{2}$.

FIGURE 3 As in 2 but for $\mathrm{H}$ runs.

FIGURE 4 As in 2 but for isopycnal model runs.

It is not immediately clear from the $\Phi_{\rho}(\phi, \rho)$ contours (Figures 2 and 3) how the flow depends on depth, as in traditional $\Phi_{z}(\phi, z)$ contours. For a more direct comparison to $\Phi_{z}$, $\Phi_{\rho}$ can be mapped on to the $(\phi, z)$ plane. To do this, we invert the zonal-average density $\rho(\phi, z)$ so that for a given $\phi$ and $\rho$ we can assign a corresponding $z$. The resulting streamlines (Figure 5, upper panels) look like traditional z-coordinate overturning, but unlike a $\Phi_{z}(\rho, z)$ figure, the flow perpendicular to an isopycnal gives a true measure of the cross-isopycnal flow. The apparent depth of a given $\Phi_{\rho}(\phi, \rho)$ streamline could differ somewhat from the 
actual depth of the flow if the isopycnal has a significant zonal tilt and the bulk of the flow occurs at a longitude where the isopycnal depth is different from its zonal mean.

FIGURE 5 Overturning streamfunction as a function of depth and latitude for integrations performed at constant- $\sigma_{2}$ (upper panels) and constant- $z$ (lower panels) for GM (left panels) and $\mathrm{H}$ (right panels) experiments. Both experiments have high $\kappa_{V}$ and high $\tau$. Contour interval is $1 \mathrm{~Sv}$. In upper panels, shading shows zonal average $\sigma_{2}$, with nonuniform contour intervals chosen to show details in both deep and shallow water. Contours are defined by making the transformation $s \equiv\left(\left[\sigma_{M}-\sigma_{2}\right] /\left[\sigma_{M}-\sigma_{m}\right]\right)^{1 / 2}$ and setting a contour interval of .05 for $s$.

Comparison of $\Phi_{z}(\phi, z)$ and $\Phi_{\rho}(\phi, z)$ for the GM and $\mathrm{H}$ experiments with high $\kappa_{V}$ and high $\tau$ (Figure 5) shows an NADW cell in all versions. The depth and strength of the volume transport is similar for $\Phi_{\rho}(\rho, z)$ and $\Phi_{z}(\rho, z)$, but other features vary. Within about $10^{\circ}$ of the northern boundary, $\Phi_{z}$ shows a much stronger maximum than $\Phi_{\rho}$, especially in the GM experiments. As described above, there is not much sign of a Deacon Cell in the GM experiments or in $\Phi_{\rho}$ for the $\mathrm{H}$ experiment, but in the $\mathrm{H}$ experiment, $\Phi_{z}$ does have a prominant Deacon Cell. Figure 5 also reveals differences between GM and H experiments that are not apparent in density coordinates. The NADW cell is confined to the top half of the water column in the GM experiment but reaches the bottom in the northern hemisphere in the $\mathrm{H}$ experiment. As we might expect, the shallower NADW cell of the GM experiment is accompanied by a more prominant AABW cell. For the isopycnal model experiments, $\Phi_{\rho}(\phi, z)$ (Figure 6) shows that the NADW cell takes up more of the water column than in the GM case, with the AABW cell correspondingly reduced. This is probably due to the layer model replacing separate temperature and salinity fields with a single $\sigma_{0}$ field. The temperature and salinity of the AABW source water makes it denser than the NADW source water, but the density difference is at least twice as great for $\sigma_{2}$ to $\sigma_{4}$, which are the appropriate measures of density for AABW water in the level model, than for $\sigma_{0}$. The 
discrepancy may also be related to the layer model's low vertical resolution in the weaklystratified abyss.

FIGURE 6 Overturning streamfunction as a function of depth and latitude for isopycnal model experiment with high $\kappa_{V}$ and high $\tau$. (a) $\Phi_{\rho}$, (b) $\Phi_{z}$. Contour interval is $1 \mathrm{~Sv}$.

We characterize the strength of the NADW cell with a single volume transport representing the maximum $\Phi_{\rho}$ in a density range which excludes the Subtropical Cells. Each H experiment has overturning values about twice as great as the corresponding GM experiment (see Table 2 and Appendix), which is a consequence of the generally greater diapycnal mixing associated with horizontal diffusion (Böning et al., 1995; Veronis, 1975). Interestingly, the isopycnal model NADW cell strength is $36 \%$ to $47 \%$ larger than the corresponding GM experiment (Table 2), though one might expect the isopycnal model to have less numerical diffusion and hence a slightly smaller overturning. Comparison of Figures 5 and 6 show that the isopycnal model has greater upwelling throughout most of the deep basin-the discrepancy is not restricted to one geographical region.

Qualitatively, our H, GM, and I experiments show similar variations with wind, vertical diffusivity, and basin width (Table 2). The H experiments are less sensitive to changes in $\kappa_{V}$ than GM and I experiments. Presumably this is because the diapycnal diffusivity in the $\mathrm{H}$ experiments is equal to $\kappa_{V}$ plus a significant contribution related to $\kappa_{H}$, so that the fractional change in diapycnal diffusivity is less than the fractional change in $\kappa_{V}$. Sensitivity to windstress is different in $\mathrm{H}$ than in GM and I, but not consistently larger or smaller.

Table 1 shows calculations of the nondimensional parameters and overturning prediction described in Section 2, and Table 3 shows the model overturning values scaled by the predictions. To measure how well the scaling fits the experiments, we compare the variation in the raw data to that of the data normalized by the scaling. The unscaled NADW cell strengths (Table 2) have a standard deviation of about $40 \%$ of the average of all the experi- 
ments in the $\mathrm{H}$ series and $50 \%$ of the average for the GM series; in both cases the statistics exclude wide-basin experiments (see following paragraph). The normalized strengths have a standard deviation of $24 \%$ of the average for the $\mathrm{H}$ experiments and $8 \%$ of the average for the GM experiments (Table 3). Statistics for the layer model overturning are similar to the GM results, with the G99 scaling reducing the scatter of the NADW strength from 51\% of the average for the raw values to $7 \%$ for the scaled values (Table 3). In summary, the scaling collapses the data for both horizontal and isopycnal/Gent-McWilliams mixing, but it provides a much better fit to the GM case.

To save on computer time, wide-basin experiments are only conducted at coarse resolution, and so are not strictly comparable to the results from other experiments in Tables 2 and 3. However, from these tables we can see that in the wide basin, changing the wind makes an $\mathrm{O}(10 \mathrm{~Sv})$ change in overturning for both $\mathrm{H}$ and $\mathrm{GM}$ mixing, and that the scaling provides a good fit to the GM case but not to the $\mathrm{H}$ case.

The G99 scaling is partially based on the idea that the wind contribution to the NADW Cell is simply the Ekman transport minus an eddy transport in the Southern Ocean. Consequently raising $\kappa_{I}$ decreases the transport, as (14), (10), and (12) show. Does horizontal diffusivity $\kappa_{H}$ play an analogous role in the $\mathrm{H}$ case? We conduct a Strong Wind H experiment with $\kappa_{H}=250 \mathrm{~cm}^{2} / \mathrm{s}$ rather than $1000 \mathrm{~cm}^{2} / \mathrm{s}$ to answer this question. The experiment is run at coarse resolution, with $\kappa_{V}=.05 \mathrm{~cm}^{2} / \mathrm{s}$, narrow basin, and all other parameters identical to the other experiments. Reducing $\kappa_{H}$ in this way increases the NADW cell strength by $16 \%$. According to (14), for these parameters, the transport should be about $6 \%$ larger if $\kappa_{I}$ is reduced from 1000 to $250 \mathrm{~cm}^{2} / \mathrm{s}$. Thus $\kappa_{H}$ plays an analogous role to $\kappa_{I}$, but (as with other parameters) has quantitative differences from the G99 scaling. 


\subsection{Overturning Anomalies Associated with Forcing Anomalies}

The strength of the NADW cell is sensitive to both $\kappa_{V}$ and $\tau$, but the overturning anomaly associated with each is quite different.

Increasing $\kappa_{V}$ increases the low- and midlatitude upwelling and has the strongest effect in the northern hemisphere (Figure 7). Because the diapycnal diffusivity is increased, the circulation changes are associated with cross-isopycnal flow. Note that the geographic distribution of changes induced by changes in $\kappa_{V}$ can be very different from the results shown here when $\kappa_{V}$ is only changed within a restricted latitude band (Marotzke and Klinger, 2000). The $\mathrm{H}$ and I overturning anomalies (not shown) are similar to the GM anomalies, but with greater magnitudes, less noise in the deep $\Phi_{z}$, and (for $\mathrm{H}$ experiments) a deeper NADW anomaly. The $\Phi_{z}$ anomaly reveals a pattern similar to overturning in a basin with no windstress, no Channel, and surface density greater at the northern boundary than at the southern boundary. In that case, the lighter hemisphere contains a relatively shallow, "subordinate" cell above the analogue of the NADW cell (Cox, 1989; Klinger and Marotzke, 1999, among others). The Channel and windstress complicate the pattern when the full overturning (as opposed to the difference between experiments) is viewed. The subordinate cell merges with the Subtropical Cell in the Weak Wind cases (Figure 2), and is eliminated by the enlarged NADW cell in the Strong Wind cases (Figures 2 and 5).

FIGURE 7 Difference in (a) $\Phi_{\rho}$ and (b) $\Phi_{z}$ between high $\kappa_{V}$ and low $\kappa_{V}$ GM experiments. Both experiments have low $\tau$. Contour interval is $1 \mathrm{~Sv}$ for both panels.

Increasing the wind south of $30^{\circ} \mathrm{S}$ creates overturning anomalies dominated by a basinspanning cell with light water flowing north and dense water flowing south (Figures 8). Most of the anomalous cross-isopycnal flow occurs near the surface. The anomalous circulation is about the same for the high $\kappa_{V}$ case as for the low $\kappa_{V}$ case (not shown). Since the wind 
anomaly is confined to the region south of $30^{\circ} \mathrm{S}$, most of the anomalous density transformation occurs there as well as north of $30^{\circ} \mathrm{N}$, where the affected isopycnals outcrop. The overturning pattern shows some asymmetry with respect to the equator; this is particularly evident in the $z$-coordinate view, in which the northward flow anomaly is confined to the Ekman layer (that is, the top level) in the southern hemisphere but spans the top $400 \mathrm{~m}$ in the northern hemisphere. H and layer model cases (not shown) are similar, but with larger and (for $\mathrm{H}$ only) deeper volume transport anomalies.

FIGURE 8 Difference in $\Phi_{\rho}$ (top panels) and in $\Phi_{z}$ (bottom panels) between Strong Wind and Weak Wind GM experiments (left panels) and Channel Wind and Weak Wind experiments (right panels). Contour interval is .5 Sv in all panels.

If the wind-driven enhancement to the NADW cell is purely a consequence of the zonal periodicity of the Channel, wind anomalies immediately north of the Channel should not affect the large-scale NADW cell strength. This is tested with the Channel Wind experiment (see Section 3). As Figure 8 shows, the anomaly is somewhat weaker than the Strong Wind anomaly. The difference in overturning between Strong Wind and Channel Wind experiments (Figure 9) shows that the wind north of the Channel is associated with an overturning cell that stretches across the basin to the northern boundary, with the Ekman flow continuing northward in the upper $500 \mathrm{~m}$ (presumably as geostrophic flow) and returning principally between $500 \mathrm{~m}$ and $1500 \mathrm{~m}$. The layer model shows a similar result, but with Ekman return flow somewhat deeper $(1-3 \mathrm{~km})$ and cross-equatorial flow somewhat larger. The Strongminus-Channel overturning difference is less isopycnal than the differences between either Strong or Channel Wind experiments and the Weak Wind experiments.

FIGURE 9 Difference in $\Phi_{\rho}$ (top panels) and in $\Phi_{z}$ (bottom panels) between Strong Wind and Channel Wind GM experiments (left panels) and between Strong Wind and Northern Wind experiments (right panels). Contour interval is .5 $\mathrm{Sv}$ in all panels. 
Comparison of the Channel Wind, Strong Wind, and Weak Wind experiments indicate that a windstress perturbation in one hemisphere of a two-hemisphere ocean leads to basinwide overturning anomaly even when the perturbation is not in a latitude belt in which the basin is zonally periodic. To further explore this behavior, we conduct the Northern Wind experiment, in which the winds north of $30 \mathrm{~N}$ are enhanced relative to the Strong Winds case (see Section 3 and Figure 1). The overturning anomaly induced by this wind anomaly is similar to the Channel-minus-Strong anomaly (Figure 9). Both show about $1 \mathrm{~Sv}$ or so of overturning anomaly reaching the equator, and both have anomalous cells confined to the top $1.5 \mathrm{~km}$ or so of the water column. These similarities exist despite the difference in the wind perturbations and the difference in which hemisphere is being perturbed. Dynamically, the northern hemisphere differs from the southern not only in geometry but also in having the sinking limb of the NADW cell. In the layer model, the Strong-minus-North overturning is somewhat weaker and does not show near antisymmetry with the Strong-minus-Channel anomaly.

For a clearer measure of how meridional volume transport anomaly varies with latitude, we take the difference in $\Phi_{z}(\phi, z)$ between two runs and then find the maximum value at each $\phi$. We calculate this for both total overturning and for the advective component only; for a given pair of experiments, we compare them to the Ekman volume transport anomaly implied by the difference in windstress between the two experiments (Figure 10). For all the pairs of experiments shown, the advective volume transport anomaly is close to that predicted by Ekman theory in the region of strongest wind anomaly. In these regions, the eddy component associated with the GM mixing tends to counteract the wind-induced transport, so that total overturning anomaly is significantly less than the advective component. Away from the wind perturbation regions, all pairs of runs have significant volume transport anomalies (both total and advective components). 
FIGURE 10 Meridional volume transport $\Phi_{z}(\phi)$ anomaly (as measured by the maximum at each latitude) for advective plus eddy component of velocity (solid line) and for advective velocity only (dashed line), GM experiments. Ekman transport predicted by difference in windstress is also shown (dotted line). (a) Strong Wind minus Weak Wind runs. (b) Channel Wind minus Weak Wind runs (dark) and Strong Wind minus Channel Wind runs (light). (c) Strong Wind minus Northern Wind runs.

How strongly the windstress just north of the Channel influences volume transport throughout the basin appears somewhat different depending on exactly which runs are compared. The volume transport anomaly relative to the Weak Wind experiment is about .5 $\mathrm{Sv}$ greater at the equator in the Strong Wind run than in the Channel Wind run (Figure 10a,b). However, the difference between the Strong Wind and Channel wind runs shows about $1 \mathrm{~Sv}$ at the equator. This is because the streamfunction anomaly associated with the wind north of the Channel is not as deep as the anomaly associated with the wind south of the Channel. In $\Phi_{\rho}$, the difference between Strong-minus-Weak and Channel-minus-Weak is also somewhat greater, with the former about $50 \%$ greater than the latter at the equator.

\subsection{Heat Transport Anomalies Associated with Forcing Anomalies}

The key contributors to the large scale meridional heat transport are the shallow Subtropical Cells driven by the tropical easterlies, the meridional overturning associated with vertical mixing (principally the NADW cell as well as a shallower cell in the southern hemisphere), and the perturbation to the NADW cell caused by the midlatitude westerly winds. By comparing the different experiments, we can analyze the contribution of each mechanism. After reviewing the other mechanisms, we will focus on the effects of the westerlies.

In the discussion below, heat transport includes advection, horizontal diffusion, and bolus velocity terms; except at high latitudes it is dominated by advection. The I experiments do not calculate separate $S$ and $T$ fields but only $\rho$, so that strictly speaking only the 
density transport, rather than the heat transport, can be calculated. To approximate the heat transport, we convert $\rho$ of each layer in the model to an equivalent $T$ based on the surface forcing: we invert the surface restoring density $\sigma_{0 R}=\sigma_{0}\left(T_{R}, S_{R}\right)$ to get $T\left(\sigma_{0}\right)$. This conversion is equivalent to assuming that if the I experiments were forced with $T$ and $S$ rather than simply $\sigma_{0}$, the $T$-S relationship throughout the domain would be the same as it is in the restoring profiles. Looking at a GM experiment (weak wind, high $\kappa_{V}$ ), this turns out to be an excellent approximation throughout most of the domain. The one exception is the upper tropical thermocline, where strong upwelling and mixing distorts this relationship somewhat; the approximation is still pretty good there as well.

In all experiments, there is at least some poleward heat transport in both hemispheres (Figure 11). In most of the experiments, the heat transport is much greater in the northern hemisphere than in the southern, reflecting the equatorial asymmetry of the NADW Cell. Despite their relatively large volume transports, the H experiments (Figure 11a) transport about the same amount of heat as the GM experiments (Figure 11b). This is partly because most of the difference in overturning occurs close to where the water sinks (Figure 5). The isopycnal experiments have peak heat transports roughly $30 \%$ bigger than GM (Figure 11c), which is commensurate with the basin-wide differences in overturning transport.

FIGURE 11 Meridional heat transport as a function of latitude for (a) GM experiments, (b) $\mathrm{H}$ experiments, and (c) layer model experiments. Each figure shows Strong Wind (solid lines), Weak Wind (dashed lines), high $\kappa_{V}$ (black) and low $\kappa_{V}$ (gray).

The Subtropical Cells are highlighted by the Weak Wind, Low- $\kappa_{V}$ experiment (Figure 2), in which all the other overturning circulations are weak. The $\Phi_{z}$ streamlines (Figure 5) suggest a simple expression for heat transport based on the Ekman transport and the surface temperature distribution; this is discussed in Klinger and Marotzke (2000). As they point out, this expression ignores contributions to the Subtropical Cell heat transport due 
to subduction poleward of the zero-windstress line $\left(30^{\circ}\right.$ latitude in our experiments and in the real ocean). This contribution is associated with the warm western boundary currents in the subtropical gyres; it is invisible in $\Phi_{z}$ but can be seen as a flow across latitude $30^{\circ}$ in $\Phi_{\rho}$ (Figure 2). Since Klinger and Marotzke (2000) discuss the Subtropical Cell in detail, we will not discuss details of the calculations here, but for our Weak Wind, Low $\kappa_{V}$ experiment, the near-equator peaks in poleward heat transport in both hemispheres (Figure 11a) are approximated by by the sum of about .1 PW predicted by the theory, about .1 PW crossing the zero-windstress line from higher latitudes, and a residual due primarily to the weak NADW cell.

When $\kappa_{V}$ is increased, the additional meridional heat transport is poleward over most of the domain in both hemispheres (Figure 12). The change is stronger in the northern hemisphere, where the thermohaline overturning is stronger, than in the southern hemisphere. Anomalies due to $\kappa_{V}$ are fairly insensitive to $\tau$ and are similar in corresponding GM, H, and I runs.

FIGURE 12 Differences in heat transport for GM experiments due to changes in $\tau$ south of $30 \mathrm{~S}$ (black solid), $\kappa_{V}$ (dashed), $\tau$ over the channel only (dash-dot) and $\tau$ north of $30 \mathrm{~N}$ (gray solid).

The heat transport associated with the classical diffusively-driven, single-hemisphere overturning has also been discussed in the context of box models (for instance, Nakamura et al., 1994; Marotzke and Stone, 1995) Poleward heat transport is approximately given by

$$
Q(\phi)=.5 c_{p} \rho \Phi_{\max }(\phi)\left(T_{S}(\phi)-T_{S}\left(\phi_{0}\right)\right)
$$

where $c_{p}=4.1 \times 10^{3} \mathrm{~J} \mathrm{~kg}^{-1} \mathrm{C}^{-1}$ is the specific heat, $\rho=1025 \mathrm{~kg} / \mathrm{m}^{3}$ is a typical density, $T_{S}$ is the surface temperature and $\phi_{0}$ is the latitude where the water sinks. The case of two hemispheres is more complicated, and it is difficult to derive a simple expression for the heat transport in the southern hemisphere, where both the "subordinate" and NADW cells 
are active. The change in northern hemisphere heat transport (Figure 12) is approximately accounted for by (20). Again, we omit details of this calculation.

Increasing the wind south of $30^{\circ} \mathrm{S}$ drives a northward heat transport anomaly at almost all latitudes (Figure 12). The anomalous density transformation poleward of latitude $30^{\circ}$ (see previous subsection) is reflected in the heat transport anomaly (Figure 12), which shows large gradients (indicating surface heat flux) there.

Wind anomalies outside the Channel have a more pronounced influence on the meridional heat transport than on the volume transport. The Channel Wind heat transport anomaly (relative to Low Wind case) is only about half as strong as the Strong Wind heat transport anomaly, while the Northern Wind heat transport anomaly (relative to Strong Wind case) is of similar magnitude to the Channel Wind anomaly (Figure 11). We can estimate the heat transport anomaly from the temperature and volume transport scales involved. If the overturning circulation anomaly has characteristic volume transport $\Phi_{A}$ and characteristic temperature difference $\Delta T$ between northward and southward-flowing limbs of the cell, then the heat transport $Q$ is given by

$$
Q=c_{p} \rho \Delta T \Phi_{A}
$$

In the previous subsection, we discussed the variations in $\Phi$ caused by the wind, but we also need to estimate $\Delta T$. If the subsurface temperature changes of a water parcel are small compared to the temperature changes at the surface, then $\Delta T$ should be given by the temperature difference between the water upwelling into the Ekman layer and the water downwelling from the Ekman layer (Klinger and Marotzke, 2000). To put it another way, the heat transport at the equator due to windstress far to the south is due to northern flow of warm water with temperature characteristic of Ekman downwelling north of the Channel and to southern flow of cold water with a temperature characteristic of Ekman upwelling 
south of the Channel. Here we approximate the average downwelling temperature by the restoring temperature at the latitude midway between the maximum and zero values of Ekman transport anomaly. For the Strong Wind case, that temperature difference is given by the SST difference between the southern edge of the Channel and 40 S. For the Channel Wind case, the water should downwell at $48 \mathrm{~S}$ instead of $30 \mathrm{~S}$. We can even include the Northern Wind case, by simply taking the temperature difference between $64 \mathrm{~N}$ and $38 \mathrm{~N}$.

Table 4 compares estimates to actual values of equatorial heat transport anomaly for each experiment. $\Phi_{A}$ values are taken from $\Phi_{z}$ differences from the numerical experiment, so the accuracy of the estimate is really a test of the sensitivity of the heat transport to the estimated temperature range. The table shows that all the heat transport estimates are within about $15 \%$ of the actual values for the numerical experiments.

\subsection{Diabatic Flow and Three-Dimensional Circulation Driven by Windstress Anomalies}

Despite the success of the above analysis in predicting the heat transport due to changes in the westerlies, it would be misleading to think that there is no diabatic circulation induced outside the density range that is ventilated in the latitude range of the wind anomaly. The streamlines in Figure 8 give the impression that all the flow pushed into the northern hemisphere by the Ekman transport anomaly dives below the surface at the northern edge of the wind anomaly. In reality, there is a surface circulation anomaly throughout the entire basin for both the Strong Wind and Channel Wind anomalies (Figure 13). In both cases, a western boundary current anomaly flows from about $30 \mathrm{~S}$ to the basin's northern boundary. For the Channel Wind anomaly, there is an anomalous surface gyre north of the wind anomaly. This gyre is also visible in Figure 9 because there is a density difference between the northward 
western boundary flow and the southward "interior" flow, but Figure 13 shows that the western boundary flow actually continues northward through the entire basin.

FIGURE 13 Surface level (25 m depth) velocity anomaly relative to Weak Wind experiment for (a) Strong Wind and (b) Channel Wind experiments. Arrow lengths are proportional to square root of speed so that both weak and strong flows can be easily seen. Alternate grid points are removed to reduce clutter in the figure. Contours show surface level temperature with contour interval of $4 \mathrm{C}$.

Thus we see two views of the induced circulation pattern: the upper limb of the cell seems to approximately follow isopycnals, but some flow remains confined to the surface, where it can cross isopycnals under the influence of surface heating and cooling. This raises the question of how much of the flow actually follows the isopycnal path. We obtain a rough measure of this by calculating the zonal average meridional velocity anomaly $v_{A}(\phi, z)$ from $\Phi_{z}(\phi, z)$. For the Strong Wind anomaly, at $25 \mathrm{~S}, v_{A}$ is almost as large at the surface as its peak value deeper in the water column, which is at about $200 \mathrm{~m}$ depth. For the Channel Wind anomaly, at $25 \mathrm{~S}$, the surface $v_{A}$ is only about half its peak value. By $15 \mathrm{~S}$, however, the surface $v_{A}$ is about half its peak value for both runs, and at $5 \mathrm{~S}$ it has further decreased to about one third the peak value for the Strong Wind anomaly. In summary, most of the induced flow approximately follows isopycnals, but there is substantial flow that is lighter and warmer than any that was pumped down in the region of wind anomaly.

\section{Conclusions}

We have used numerical models with different parameterizations of eddy-mixing of density (horizontal (H) and isopycnal/Gent-McWilliams (GM)) and different discretizations (level and layer (I)) to study the sensitivity of an idealized Atlantic basin to vertical mixing and basin width and to wind strength over a zonal subpolar channel analogous to Earth's South- 
ern Ocean. For all versions of the experiments, increases in diapycnal mixing or subpolar windstress increase the strength of the model's version of the North Atlantic Deep Water (NADW) meridional overturning cell. There are quantitative differences between the different mixing and numerical schemes; in particular, the greater diapycnal mixing associated with horizontal mixing (compared to isopycnal mixing), makes for a larger overturning. For a given change in windstress forcing, the absolute change in overturning is larger for the $\mathrm{H}$ case than for the GM case but the fractional change in overturning is about the same size (sometimes larger, sometimes smaller, depending on other parameters). The I experiments also have larger overturning than the GM experiments, but have very similar fractional changes in overturning when the forcing and basin width are changed.

Gnanadesikan (1999, here denoted G99) derived a cubic equation which describes the strength of the NADW cell. We nondimensionalize this cubic and derive a simplified, closed-form expression for the resulting scaling. The expression relates the pycnocline depth and overturning strength to the classical advective-diffusive scales for these quantities and to nondimensional external parameters representing the Ekman transport and thickness diffusion in the Southern Ocean. The relative importance of the windstress and vertical mixing for driving deep meridional overturning in the real world depends on some largescale average of vertical eddy diffusivity, $\kappa_{V}$, which is difficult to measure. Our analysis of the scaling implies that the windstress contribution could at least double the overturning (compared to the overturning driven by mixing alone) if $\kappa_{V}$ is less than about $.1-.2 \mathrm{~cm}^{2} / \mathrm{s}$.

The G99 scaling only roughly fits the $\mathrm{H}$ experiments but provides an excellent fit to the experiments with isopycnal/GM mixing (experiments GM and I).

We show that enhanced Southern Ocean westerlies drive an overturning anomaly in the form of a basin-wide cell connecting Southern Ocean upwelling and downwelling near the 
northern boundary of the basin, consistent with Togweiler and Samuels' (1995) conclusions based on total (as opposed to anomaly) overturning. The anomalies may be relevant to oceanic variability, since variability in the mean westerlies may drive similar anomalies in the ocean. Windstress fluctuations, as a fraction of the time-mean, are likely to be weaker than is imposed in our experiments, but the wider zonal extent of the real Southern Ocean might allow such wind-induced overturning variability to be measurable.

Despite the success of the G99 scaling, several factors controlling the NADW cell strength are outside the scope of the G99 scaling. In particular, the G99 scaling does not take into account wind outside the subpolar channel. We find that this component affects the basin-wide volume transport. Moreover, Tsujino and Suginohara (1999) find that windstress applied to one hemisphere of a basin with no channel also has a strong effect on the deep overturning cell. This result complicates the original picture of Toggweiler and Samuels (1995) which argues that the basin-wide influence of the subpolar winds is due to the presence of a channel which makes it harder for equatorward Ekman transport in the channel to downwell locally. In our experiments, wind perturbations just north of the channel or north of $30 \mathrm{~N}$ produce basin-wide circulation anomalies that are shallower than anomalies induced by similar windstress perturbations in the Channel, with volume transport about $1 / 3$ as strong and heat transport anomalies about half as strong. The experiments of Tsujino and Suginohara (1999) imply that wind-forcing outside the Channel latitudes should drive a stronger remote overturning anomaly then we find here, with less of the subpolar meridional Ekman transport returned locally and more making the basin-wide circuit. We analyze the differences between their experiments and ours in an accompanying paper.

Previous studies have explained how the magnitude of heat transport follows from the overturning circulation associated with the trade winds and the mixing-driven NADW cell. Here we separate the total heat transport into components associated with these processes as 
well as a component due to midlatitude westerlies. We then estimate the heat transport due to the westerlies based on the volume transport anomaly and externally-imposed surface temperature. By assuming that the anomalous Ekman pumping which flows across the equator follows an approximately isopycnal path, we make fairly accurate predictions of the heat transport driven by wind both inside the Channel latitudes and outside. However, closer examination of the velocity anomaly shows that there is also a surface western boundary current anomaly flowing from the wind perturbation region to the equator and beyond. In our experiments, this part of the flow is relatively small. However, it is not clear what controls the proportion of the cross-equatorial flow that follows the surface path, and this proportion may be different in different experimental configurations, such as one with a more intense (and realistic) western boundary current.

\section{Appendix: Resolution Sensitivity}

In many evaluations of resolution in general circulation models, reductions in grid-spacing are accompanied by reductions in viscosity, so that the western boundary current (WBC) width decreases with gridspacing. Here we maintain the same viscosity so that we can focus purely on the convergence of the numerical approximation to the continuum equations of motion. As Table 5 shows, when the channel numerical experiments described above are performed with $4^{\circ} \times 3.75^{\circ}$ resolution, the overturning strengths are somewhat higher than with $2^{\circ} \times 2^{\circ}$ resolution experiments. The $\mathrm{H}$ experiments are much more sensitive to resolution than the GM experiments. For the layer model, the sensitivity to resolution is higher for weak diapcynal mixing. In that case, the watermass transformation is dominated by buoyancy change at the surface, which is treated differently in the layer model. Note that all parameters are identical in corresponding high and low resolution experiments. A velocity section through the WBC of the high- $\kappa_{V}$, low-wind GM case shows that the current is the 
same width at both resolutions, indicating that even at coarse resolution the grid is resolving the WBC. Nevertheless, only about two gridpoints are included in the WBC at coarse resolution. This may be especially problematic because of the flux-corrected transport scheme we use, which can introduce a significant amount of numerical diffusivity in temperature structures that are just barely resolved by the grid (Griffies et al., 2000).

Further high- $\kappa_{V}$, low-wind experiments at $1^{\circ} \times 1^{\circ}$ resolution improve our knowledge of how well the numerical experiments approximate the continuous problem (see Table 6). Linearly extrapolating the $2^{\circ}$ and $1^{\circ}$ experiments down to 0 gridspacing, we can estimate the "infinite resolution" solution as well as the error in overturning in each of the finite resolution experiments (Table 6). Extrapolation is always a somewhat speculative enterprise; the overturning is a linear function of grid spacing for $\mathrm{H}$ experiments but for $\mathrm{GM}$ experiments it is less sensitive at smaller grid spacing. The results show that for GM mixing, even $4^{\circ} \times 3.75^{\circ}$ resolution gives overturning which is less than $10 \%$ greater than the estimated infinite resolution solution. In $\mathrm{H}$ mixing experiments, numerical errors are leading order at $4^{\circ} \times 3.75^{\circ}$, and even $1^{\circ}$ resolution may have significant errors. At all resolutions, the $\mathrm{H}$ experiment has greater NADW cell volume transport than the corresponding GM experiment. While GM experiments take about twice as much computer time as corresponding $\mathrm{H}$ experiments, for a given WBC width, the GM parameterization may actually demand less computer time because not as high horizontal resolution is required.

The ratio of $\mathrm{H}$ overturning to GM overturning increases linearly with gridspacing. We extrapolate the ratio down to $0^{\circ}$ gridspacing. At $3.75^{\circ}$, the overturning is 1.8 to 2.5 times greater for $\mathrm{H}$ than for corresponding GM experiments. For the $0^{\circ}$ extrapolation, $\mathrm{H}$ is only 1.1 to 1.7 times greater than GM (less than 1.5 for high $\kappa_{V}$, more than 1.5 for low $\kappa_{V}$ ). 


\section{Acknowledgements}

Barry Klinger was supported by NSF grant OCE-9730554 at NSU and OCE-0196117 at GMU. Elena Boguslavsky and Kevin Kohler gave computer assistance. Thanks go to Paul Schopf for some interesting discussion. 


\section{References}

Arakawa, A., and V. Lamb, 1977: Computational design of the basic dynamical prosecces of the UCLA general circulation model. Methods in Computational Physics, 17, 174-267.

Bleck, R., and D. B. Boudra, 1986: Wind driven spin-up in eddy-resolving ocean models formulated in isopycnic and isobaric coordinates. J. Geophys. Res., 91, 7611-7621.

- , and L. T. Smith, 1990: A wind-driven isopycnic coordinate model of the North and Equatorial Atlantic Ocean. 1. Model development and supporting experiments. J. Geophys. Res., 95, 3273-3285.

Bo"ning, C.W., W.R. Holland, F.O. Bryan, G. Danabasoglu, and J.C. McWilliams. An overlooked problem in model simulations of the thermohaline circulation and heat transport in the Atlantic Ocean, J. Clim., 8, 515-523, 1995.

Bryan, F., 1987: Parameter sensitivity of primitive equation ocean general circulation models. J. Phys. Oceanogr., 17, 970-985.

Bryan, K., 1984: Accelerating the convergence to equilibrium of ocean-climate models, $J$. Phys. Oceanogr., 14, 666-673.

Bryan, K., and M. D. Cox, 1967: A numerical investigation of the oceanic general circulation. Tellus, 19, 54-80.

Colin de Verdiere, A., 1988: Buoyancy driven planetary flows. J. Mar. Res., 46, 215-265.

Cox, M. D., 1989, An idealized model of the world ocean. Part I: The global-scale water masses. J. Phys. Oceanogr., 19, 1730-1752.

Danabasoglu, G., J. C. McWilliams, and P. R. Gent, 1994: The role of mesoscale tracer transports in the global ocean circulation. Science, 264, 1123-1126. 
Danabasoglu, G., and J. C. McWilliams, 1995: Sensitivity of the global ocean circulation to parameterization of mesoscale tracer transports. J. Climate, 8, 2967-2987.

Doos, K, and D. J. Webb, 1994: The Deacon Cell and the other meridional cells in the Southern Ocean, J. Phys. Oceanogr., 24, 429-442.

Drijfhout, S. S., 1994: On the heat transport by mesoscale eddies in an ocean circulation model. J. Phys.

Oceanogr., 24, 429-442.

Gent, P. R., and J. C. McWilliams, 1990: Isopycnal mixing in ocean circulation models. J. Phys. Oceanogr., 20, 150-155.

Gerdes, R., C. Koberle, and J. Willebrand, 1991: The influence of numerical advection schemes on the results of ocean general circulation models, Climate Dyn., 5, 211-226.

Gnanadesikan, A, 1999: A simple predictive model for the structure of the oceanic pycnocline, Science, 283, 2077-2079.

Gregg, 1987: Diapycnal mixing in the thermocline: a review. J. Geophys. Res., 92, 52495286.

Griffies, S. M., R. C. Pacanowski, and R. W. Hallberg, 2000: Spurious diapycnal mixing associated with advection in a z-coordinate ocean model, Monthly Weather Rev., 128, $538-564$.

Hellerman, S., and M. Rosenstein, 1983: Normal monthly wind stress over the World Ocean with error estimates, J. Phys. Oceanogr., 13, 1093-1104.

Hirst, A. C., D. R. Jackett, and T. J. McDougall, 1996: The meridional overturning cells of a World Ocean model in neutral density coordinates. J. Phys. Oceanogr., 26, 775-791 
Hirst, A. C., and T. J. McDougall, 1998: Meridional overturning and dianeutral transport in a z-coordinate ocean model including eddy-induced advection, J. Phys. Oceanogr., 28, 1205-1223.

Huang, R. X., and K. Bryan, 1987: A multilayer model of the thermohaline and wind-driven ocean circulation. J. Phys. Oceanogr., 17, 1909-1924.

Klinger, B., 2000: Acceleration of general circulation model convergence by exponential extrapolation, Ocean Modelling, 2, 61-72

Klinger, B. A., and J. Marotzke, 1999: Behavior of double hemisphere thermohaline flows in a single basin. J. Phys. Oceanogr., 29, 382-399.

Klinger, B. A., and J. Marotzke, 2000: Meridional heat transport by the subtropical cell. J. Phys. Oceanogr., 30, 696-705.

Ledwell, J. R., A. J. Watson, and C. S. Law, 1993: Evidence for slow mixing across the pycnocline from an open-ocean tracer-release experiment. Nature, 365, 701-703.

Marotzke, J., and P. H. Stone, 1995: Atmospheric transports, the thermohaline circulation, and flux adjustments in a simple coupled model. J. Phys. Oceanogr., 25, 1350-1364.

Marotzke, J., and B. A. Klinger, 2000: Dynamics of equatorially asymmetric thermohaline circulations. J. Phys. Oceanogr., 30, 950-970.

McDermott, D. A., 1996: The regulation of nothern overturning by southern hemisphere winds. J. Phys. Oceanogr., 26, 1234-1255.

McDougall, T. J., 1987: Neutral surfaces. J. Phys. Oceanogr., 17, 898-942.

McCreary, J., and P. Lu, 1994: Interaction between the subtropical and equatorial ocean circulations: the Subtropical Cell. J. Phys. Oceanogr., 24, 466-497. 
Nakamura, M., P. H. Stone, and J. Marotzke, 1994: Destabilization of the thermohaline circulation by atmospheric eddy transports. J. Climate, 7, 1870-1882.

Pacanowski, R. C., 1976: MOM 2 Version 2.0 (Beta) Documentation User's Guide and Reference Manual, GFDL Ocean Technical Report 3.2, 329 pp.

Park, Y.-G., and K. Bryan, 2000: Comparison of thermally driven circulations from a depth coordinate model and an isopycnal layer mode: Part. I. A scaling law - sensitivity to vertical diffusivity. J. Phys. Oceanogr., 30, 590-605.

Polzin, K., J. Toole, J. Ledwell, and R. Schmitt, 1997: Spatial variability of turbulent mixing in the abyssal ocean. Science, 276, 93-96.

Rahmstorf, S., and M. H. England, 1997: Influence of Southern hemisphere winds on North Atlantic Deep Water flow. J. Phys. Oceanogr., 27, 2040-2054.

Scott, J.R., 2000: The roles of geothermal heating, diapycnal mixing, and surface bouyancy forcing in meridional overturning dynamics. Ph.D. Thesis, MIT, 128 pp.

Toggweiler, J. R., and B. Samuels, 1995: Effect of Drake Passage on the global thermohalinel circulation, Deep-Sea Res., 42, 477-500.

Toggweiler, and B. Samuels, 1998: On the ocean's large-scale circulation near the limit of no vertical mixing. J. Phys. Oceanogr., 28, 1832-1852.

Toole, J., R. Schmitt, K. Polzin, and E. Kunze, 1997: Fine and microstructure evidence of boundary mixing above the flanks of a mid-latitude seamount. J. Geophys. Res., 102, $947-959$.

Tsujino and Suginohara, 1999: Thermohaline circulation enhanced by wind forcing J. Phys. Oceanogr., 29, 1506-1516. 
Veronis, G., 1975: The role of models in tracer studies. Numerical Models of the Ocean Circulation. National Academy of Sciences, 133-146.

Visbeck, M., J. Marshall, T. Haine, and M. Spall, 1997: Specifiation of eddy transfer coefficients in coarse resolution ocean circulation models. J. Phys. Oceanogr., 27, $381-402$. 


\section{Figure Captions}

1. Surface forcing as a function of latitude for experiments. Restoring profiles for (a) temperature (solid, left axis labels) and salinity (dashed, right axis labels), and (b) density as measured by $\sigma_{0}$ (solid) and $\sigma_{2}$ (dashed, offset by $9.3115 \mathrm{~kg} / \mathrm{m}^{3}$ ). (c) Zonal windstress for weak wind (heavy solid), strong southern wind (solid), strong channel wind (solid with dots) and strong northern and southern wind (dashed). (d) Meridional Ekman volume transport; same linestyles as in (c).

2. Meridional overturning for low and high vertical diffusivity $\kappa_{V}$ and low and high southern wind $\tau$, GM runs including both advective and eddy components. Contour interval $=1 \mathrm{~Sv}$. Overturning with $\sigma_{2}$ coordinate is calculated using 51 unequally spaced density intervals which give higher resolution for higher $s_{i g m a}$.

3. As in 2 but for $H$ runs.

4. As in 2 but for isopycnal model runs.

5. Overturning streamfunction as a function of depth and latitude for integrations performed at constant- $\sigma_{2}$ (upper panels) and constant- $z$ (lower panels) for GM (left panels) and $\mathrm{H}$ (right panels) experiments. Both experiments have high $\kappa_{V}$ and high $\tau$. Contour interval is $1 \mathrm{~Sv}$. In upper panels, shading shows zonal average $\sigma_{2}$, with nonuniform contour intervals chosen to show details in both deep and shallow water. Contours are defined by making the transformation $s \equiv\left(\left[\sigma_{M}-\sigma_{2}\right] /\left[\sigma_{M}-\sigma_{m}\right]\right)^{1 / 2}$ and setting a contour interval of .05 for $s$.

6. Overturning streamfunction as a function of depth and latitude for isopycnal model experiment with high $\kappa_{V}$ and high $\tau$. (a) $\Phi_{\rho}$, (b) $\Phi_{z}$. Contour interval is 1 Sv. 
7. Difference in (a) $\Phi_{\rho}$ and (b) $\Phi_{z}$ between high $\kappa_{V}$ and low $\kappa_{V}$ GM experiments. Both experiments have low $\tau$. Contour interval is $1 \mathrm{~Sv}$ for both panels.

8. Difference in $\Phi_{\rho}$ (top panels) and in $\Phi_{z}$ (bottom panels) between Strong Wind and Weak Wind GM experiments (left panels) and Channel Wind and Weak Wind experiments (right panels). Contour interval is .5 $\mathrm{Sv}$ in all panels.

9. Difference in $\Phi_{\rho}$ (top panels) and in $\Phi_{z}$ (bottom panels) between Strong Wind and Channel Wind GM experiments (left panels) and between Strong Wind and Northern Wind experiments (right panels). Contour interval is .5 Sv in all panels.

10. Meridional volume transport $\Phi_{z}(\phi)$ anomaly (as measured by the maximum at each latitude) for advective plus eddy component of velocity (solid line) and for advective velocity only (dashed line), GM experiments. Ekman transport predicted by difference in windstress is also shown (dotted line). (a) Strong Wind minus Weak Wind runs. (b) Channel Wind minus Weak Wind runs (dark) and Strong Wind minus Channel Wind runs (light). (c) Strong Wind minus Northern Wind runs.

11. Meridional heat transport as a function of latitude for (a) GM experiments, (b) $\mathrm{H}$ experiments, and (c) layer model experiments. Each figure shows Strong Wind (solid lines), Weak Wind (dashed lines), high $\kappa_{V}$ (black) and low $\kappa_{V}$ (gray).

12. Differences in heat transport for GM experiments due to changes in $\tau$ south of $30 \mathrm{~S}$ (dark solid), $\kappa_{V}$ (dashed), $\tau$ over the channel only (dash-dot) and $\tau$ north of $30 \mathrm{~N}$ (gray solid).

13. Surface level (25 m depth) velocity anomaly relative to Weak Wind experiment for (a) Strong Wind and (b) Channel Wind experiments. Arrow lengths are proportional to square root of speed so that both weak and strong flows can be easily seen. Alternate 
grid points are removed to reduce clutter in the figure. Contours show surface level temperature with contour interval of $4 \mathrm{C}$. 


\section{Table 1: Scaling for Numerical Experiments}

\begin{tabular}{llll|l|l|l|l|c} 
& & & & \multicolumn{2}{c}{$E$} & \multicolumn{2}{c}{$d$} & $T_{N}$ \\
$\kappa_{V}$ & $T_{0}$ & $D_{0}$ & $S$ & lowind & hiwind & lowind hiwind & lowind hiwind \\
\hline
\end{tabular}

Default Basin

\begin{tabular}{rrrr|rr|rr|rr}
.05 & 4.1 & 91 & .15 & 0.85 & 2.6 & 1.2 & 1.7 & 5.8 & 11.2 \\
.50 & 19.0 & 195 & .069 & 0.18 & 0.55 & 1.0 & 1.1 & 20.3 & 24.5
\end{tabular}

Wide Basin

\begin{tabular}{llll|ll|ll|ll}
.05 & 10.3 & 144 & .37 & 1.4 & 4.1 & 1.3 & 2.0 & 16.3 & 39.6 \\
.50 & 48.0 & 310 & .17 & 0.3 & 0.9 & 1.0 & 1.2 & 51.2 & 68.0
\end{tabular}

$\kappa_{V}$ is in $\mathrm{cm}^{2} / \mathrm{s}, T_{0}$ and $T_{N}$ in $\mathrm{Sv}, D_{0}$ in $\mathrm{m}$, and $S, E$, and $d$ are nondimensional.

\section{Table 2: Numerical Experiments, Overturning Strength}

\begin{tabular}{r|rr|rr|rr} 
& \multicolumn{3}{|c}{ H (Level Model) } & \multicolumn{2}{c}{ GM (Level Model) } & \multicolumn{2}{c}{ Layer Model } \\
$\kappa_{V}$ & lowind & hiwind & lowind & hiwind & lowind & hiwind \\
\hline .05 & 6.6 & 10.6 & 3.1 & 5.5 & 3.9 & 7.5 \\
.50 & 13.6 & 18.4 & 9.2 & 11.1 & 11.8 & 15.1 \\
& \multicolumn{5}{|c}{ Wide Basin, Coarse Resolution } \\
.05 & 26.1 & 39.8 & 9.5 & 17.5 &
\end{tabular}

$\kappa_{V}$ as in Table 1; all other numbers refer to maximum in NADW cell overturning streamfunction $(\mathrm{Sv})$ calculated in density coordinate; for GM runs this is for total streamfunction (advective plus eddy terms).

\begin{tabular}{c|cr|rr|rr}
\multicolumn{5}{|c}{ Table 3: Scaled Overturning From Numerical Experiments } \\
& \multicolumn{2}{|c}{ H (Level Model) } & \multicolumn{3}{c}{ GM (Level Model) } & \multicolumn{2}{c}{ Layer Model } \\
$\kappa_{V}$ & lowind & hiwind & lowind & hiwind & lowind & hiwind \\
\hline .05 & 1.14 & 0.95 & 0.53 & 0.49 & 0.67 & 0.67 \\
.50 & 0.67 & 0.75 & 0.45 & 0.45 & 0.58 & 0.62 \\
& Wide Basin, Coarse Resolution & & & & \\
.05 & 1.60 & 1.01 & .58 & .44 & &
\end{tabular}

$\kappa_{V}$ as in Table 1, all other numbers are from Table 2 scaled by corresponding $T_{N}$ from Table 1 (nondimensional). 
Table 4: Estimate and Actual Equatorial Heat Transport Anomaly

\begin{tabular}{lrlll} 
Run & $\Delta T$ & $\Phi_{A}$ & $Q_{\text {est }}$ & $Q_{\text {act }}$ \\
\hline Strong - Weak & 12.3 & 2.5 & .13 & .14 \\
Channel - Weak & 7.5 & 2.0 & .063 & .072 \\
Strong - Northern & 13.5 & 1.0 & .074 & .057
\end{tabular}

$\Delta T$ in $\mathrm{C}, \Phi_{A}$ in Sv, $Q_{\text {est }}$ (estimated equatorial heat transport) and $Q_{\text {act }}$ actual equatorial heat transport in $\mathrm{PW}$.

Table 5: Comparison of Low Resolution and High Resolution Overturning

\begin{tabular}{l|rr|rr|rr}
$\kappa_{V}$ & \multicolumn{2}{|c}{ H (Level Model) } & \multicolumn{2}{c}{ GM (Level Model) } & \multicolumn{2}{c}{ Layer Model } \\
\hline .05 (ratio) & lowind & hiwind & lowind & hiwind & lowind & hiwind \\
.50 (ratio) & 1.18 & 1.34 & 1.00 & 1.15 & 1.46 & 1.35 \\
.05 (difference) & 1.30 & 1.26 & 1.07 & 1.12 & 1.12 & 1.11 \\
.50 (difference) & 4.1 & 3.6 & 0.0 & 0.8 & 1.8 & 2.6 \\
& 4.8 & 0.6 & 1.3 & 1.4 & 1.7
\end{tabular}

$\kappa_{V}$ as in Table 1. Numbers refer to NADW peak volume transport in $\rho$ coordinates; "ratio" (nondimensional) refers to low resolution divided by high resolution values, and "difference" $(\mathrm{Sv})$ is low resolution minus high resolution.

Table 6: Overturning as a Function of Resolution

\begin{tabular}{l|r|r|rrr} 
& \multicolumn{3}{|c}{ Overturning } & \multicolumn{4}{c}{ Overturning Ratio } \\
Mixing & $1^{\circ}$ & $0^{\circ}$ Est & $1^{\circ}$ & $2^{\circ}$ & $3.75^{\circ}$ \\
\hline $\mathrm{H}$ & 12.0 & 10.4 & 1.15 & 1.31 & 1.70 \\
$\mathrm{GM}$ & 9.1 & 9.0 & 1.01 & 1.02 & 1.09
\end{tabular}

Overturning is $\rho$-coordinate NADW volume transport $(\mathrm{Sv})$ for $1^{\circ}$ resolution experiments and extrapolation to $0^{\circ}$ resolution. Overturning ratio is volume transport normalized by $0^{\circ}$ estimate. 
(a) T and S

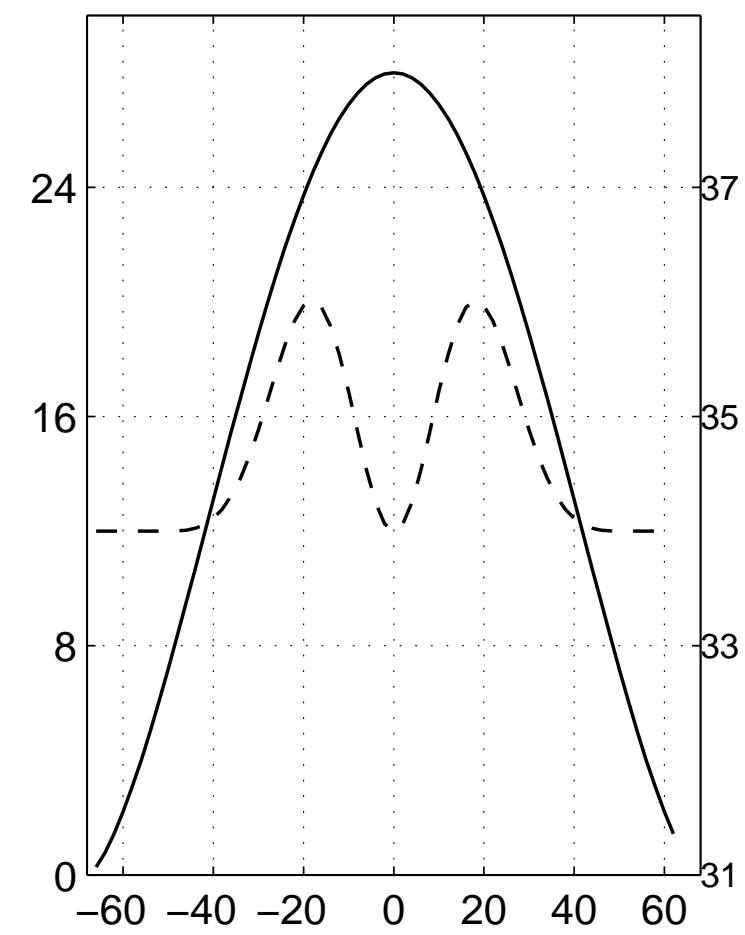

(c) windstress $\left(\mathrm{N} / \mathrm{m}^{2}\right)$

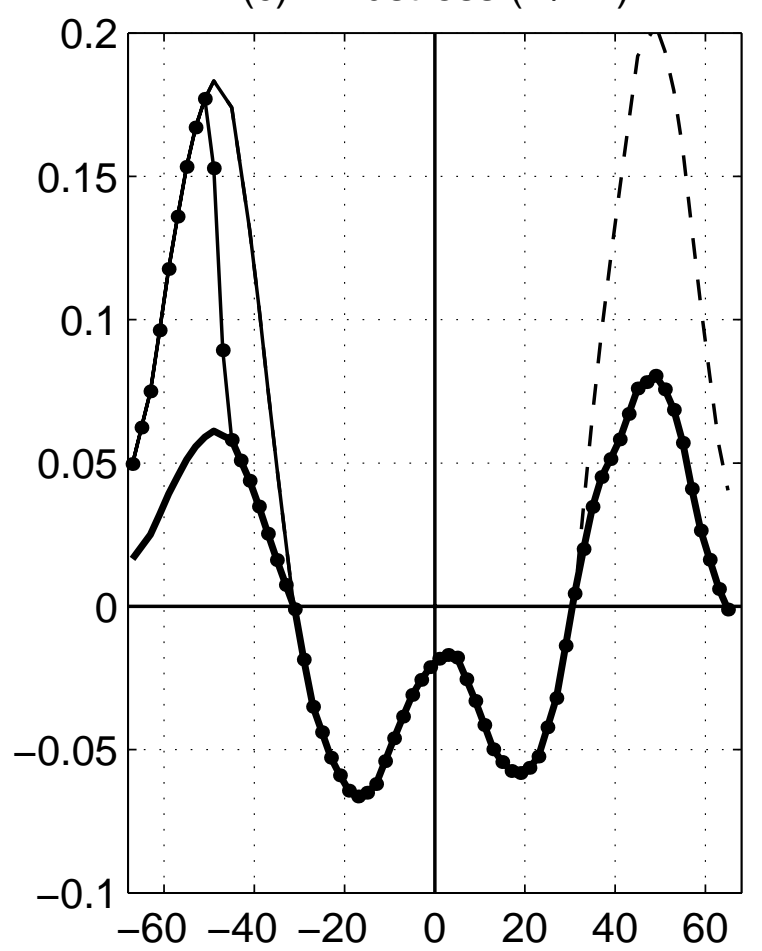

(b) $\sigma_{0}$ and $\sigma_{2}$

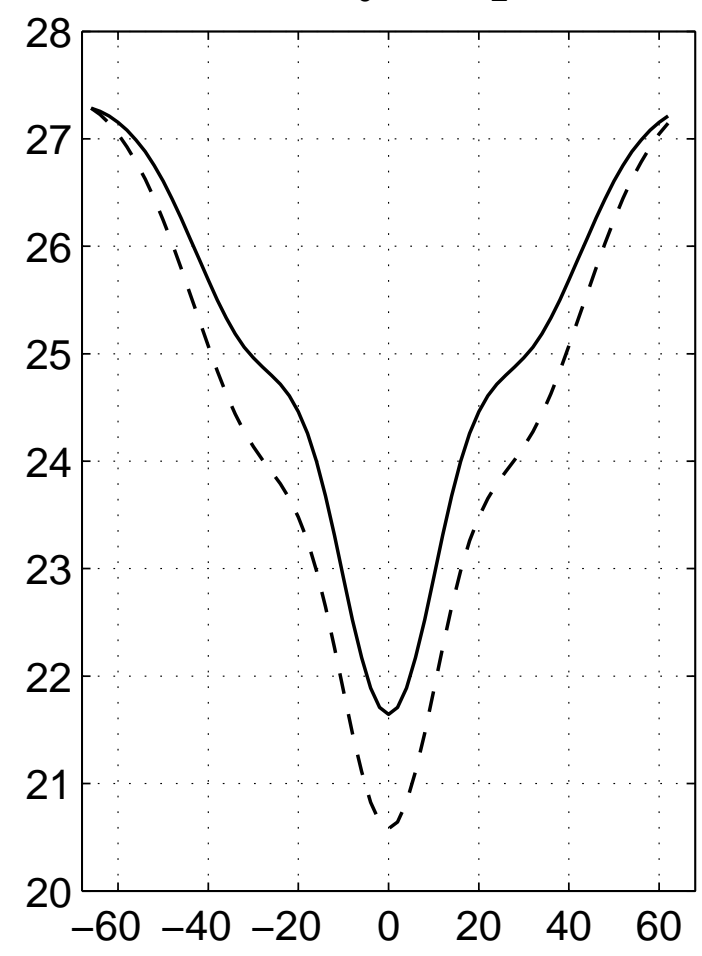

(d) Ek vol trans (Sv)

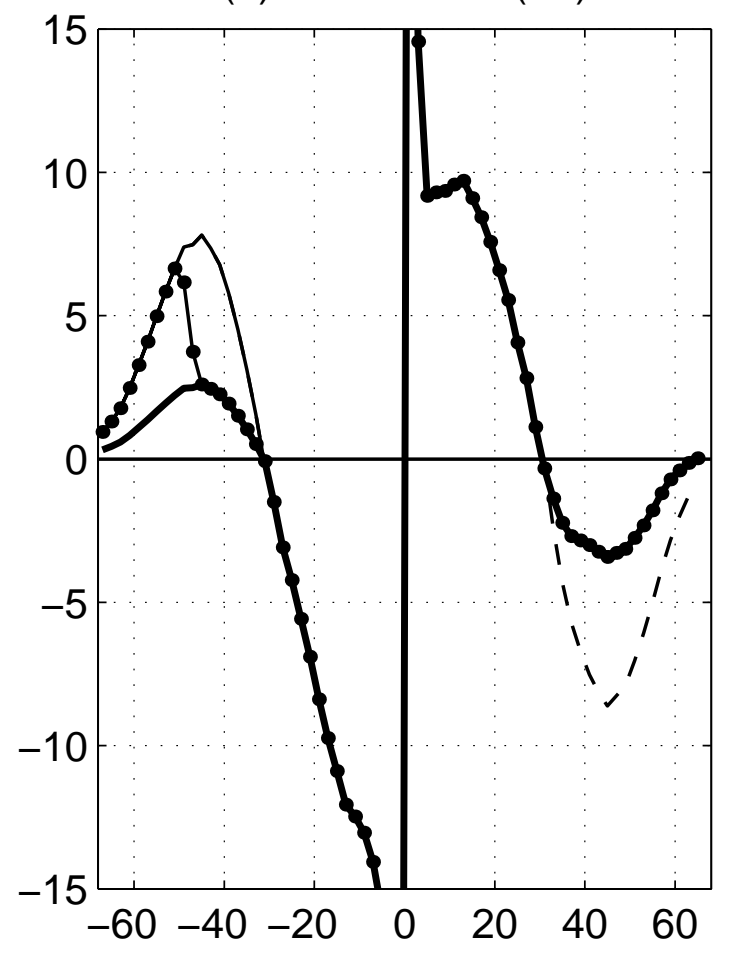



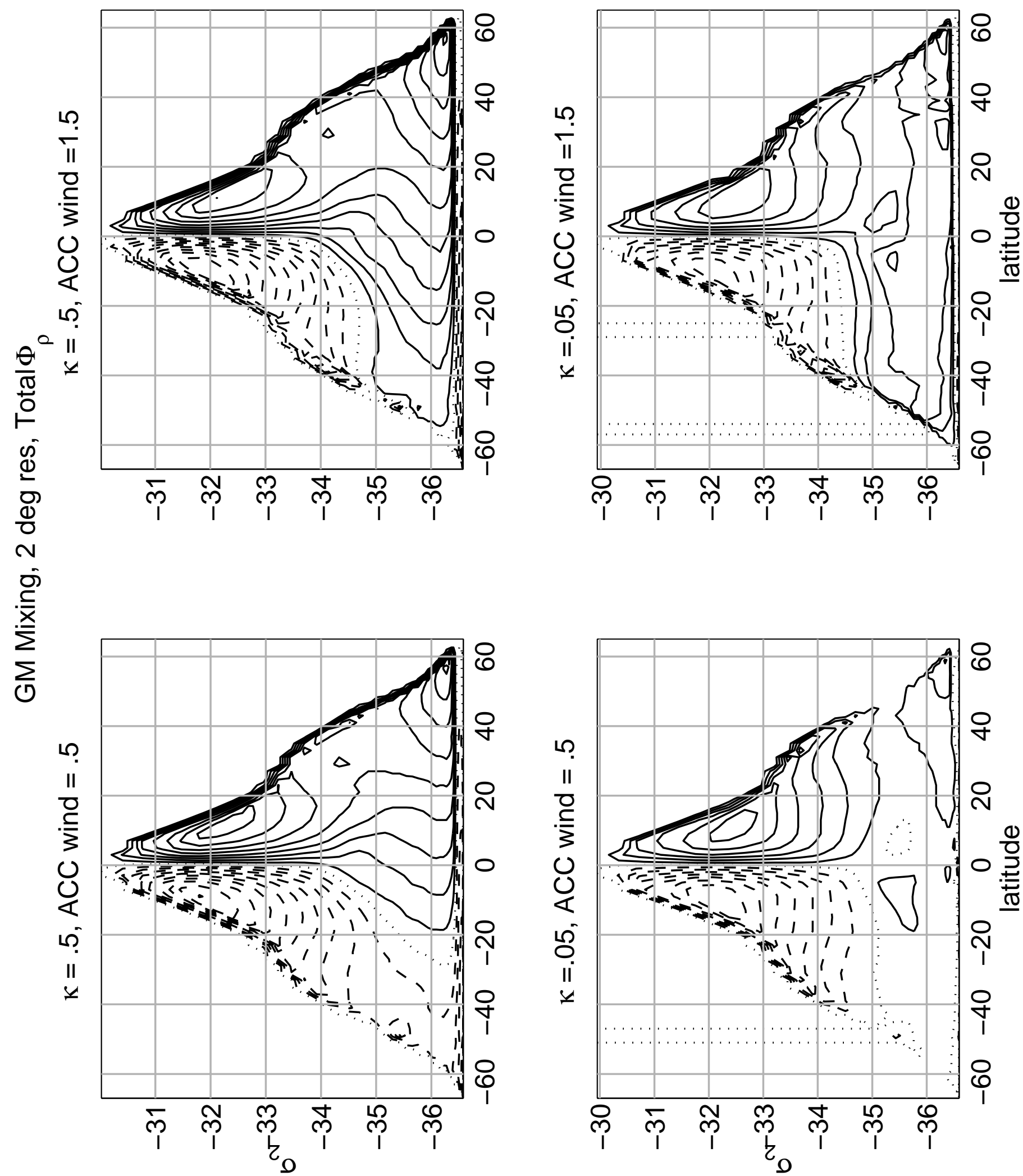

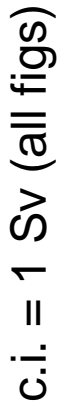

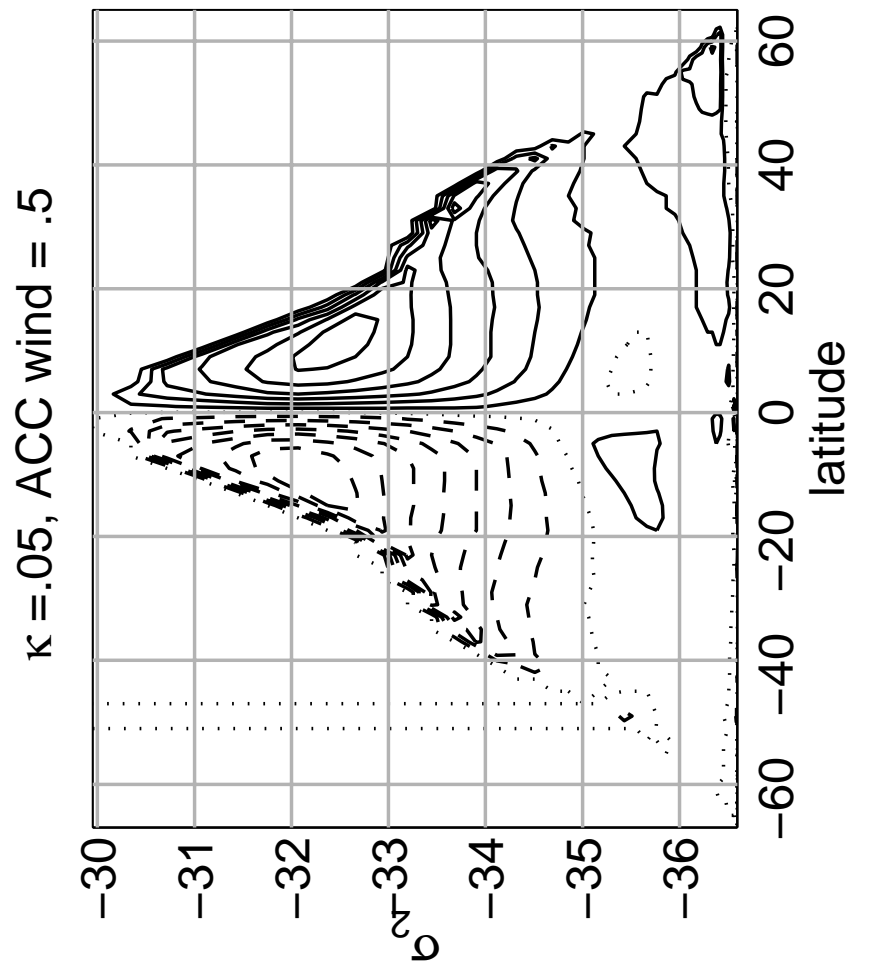



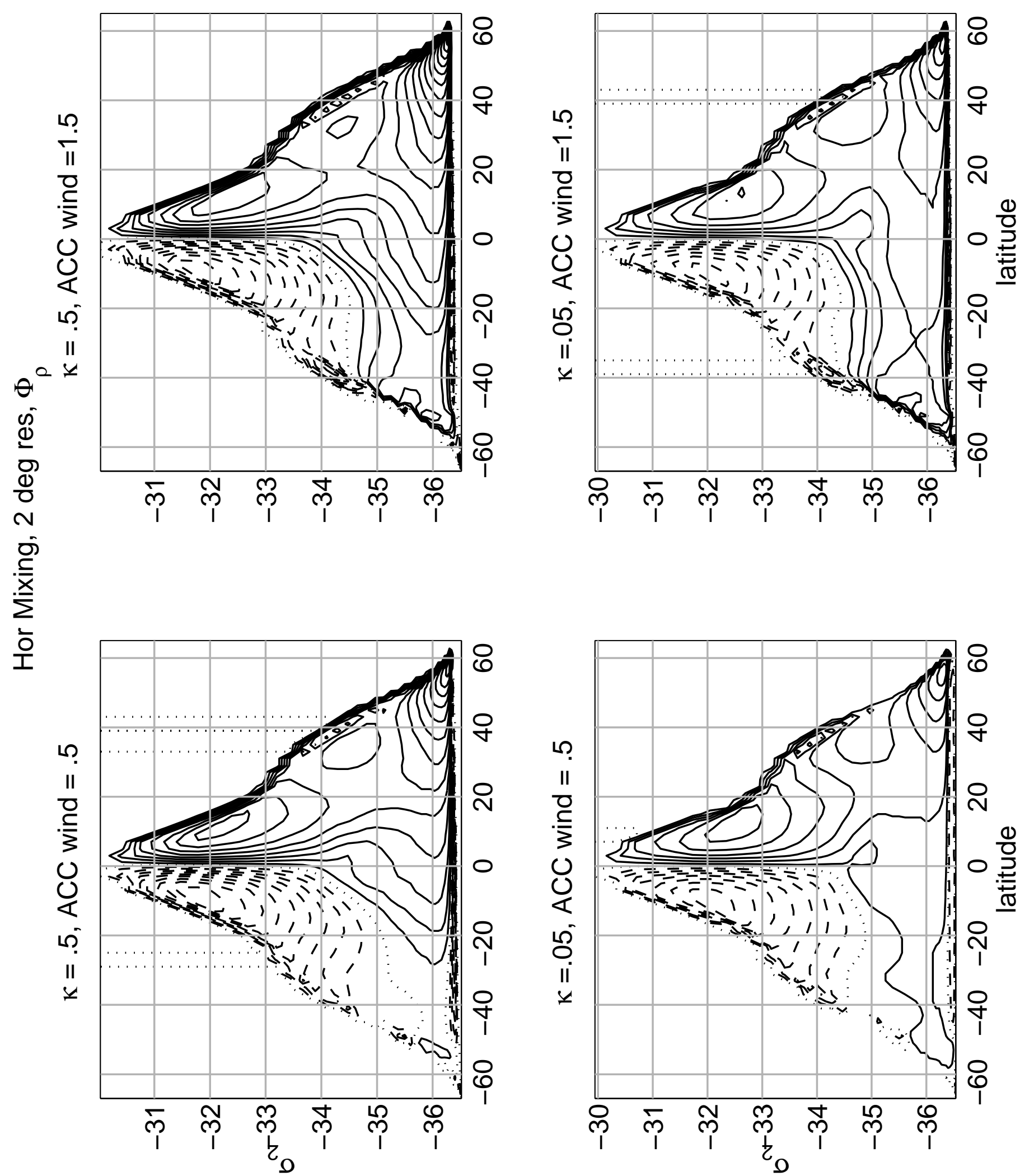

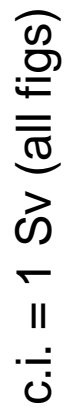

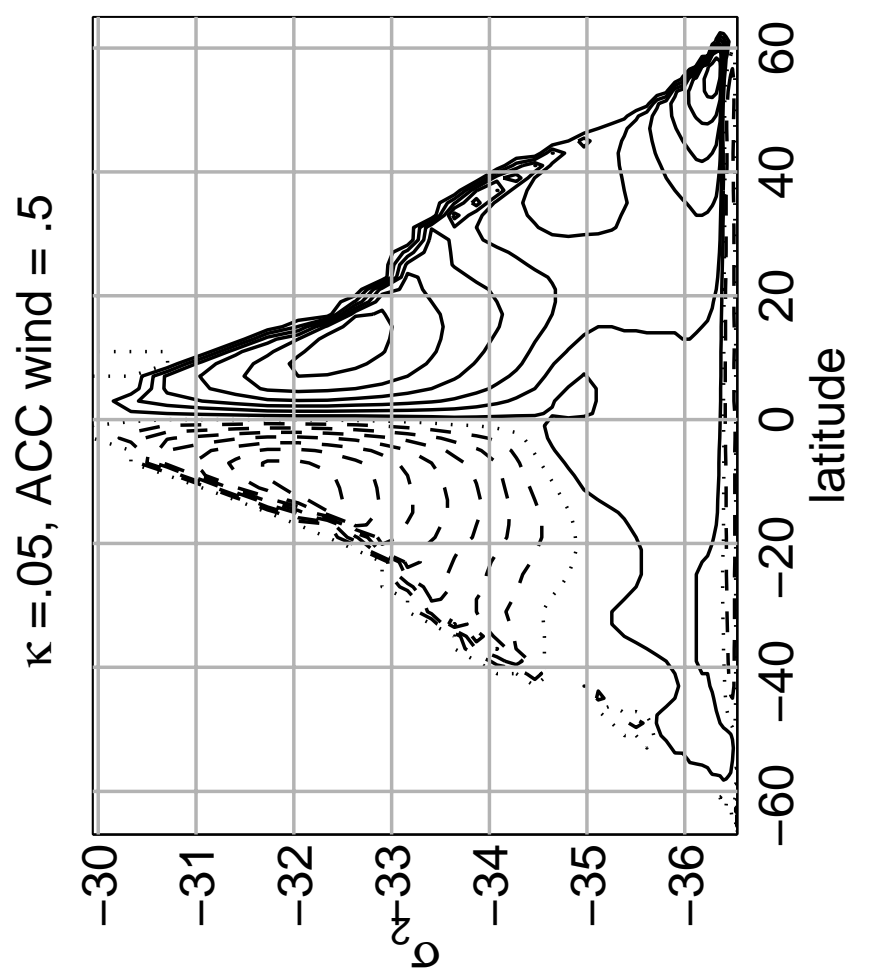




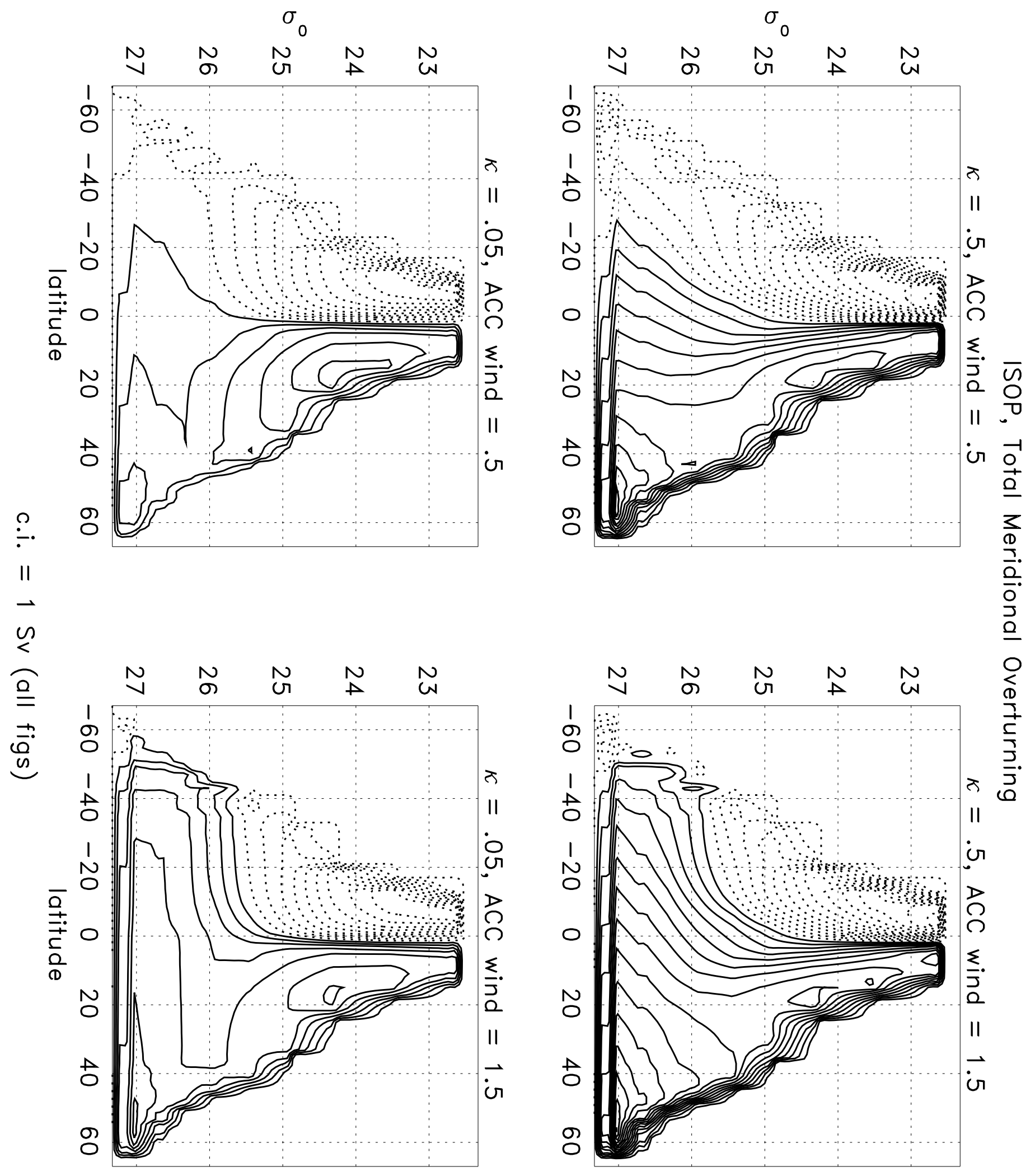



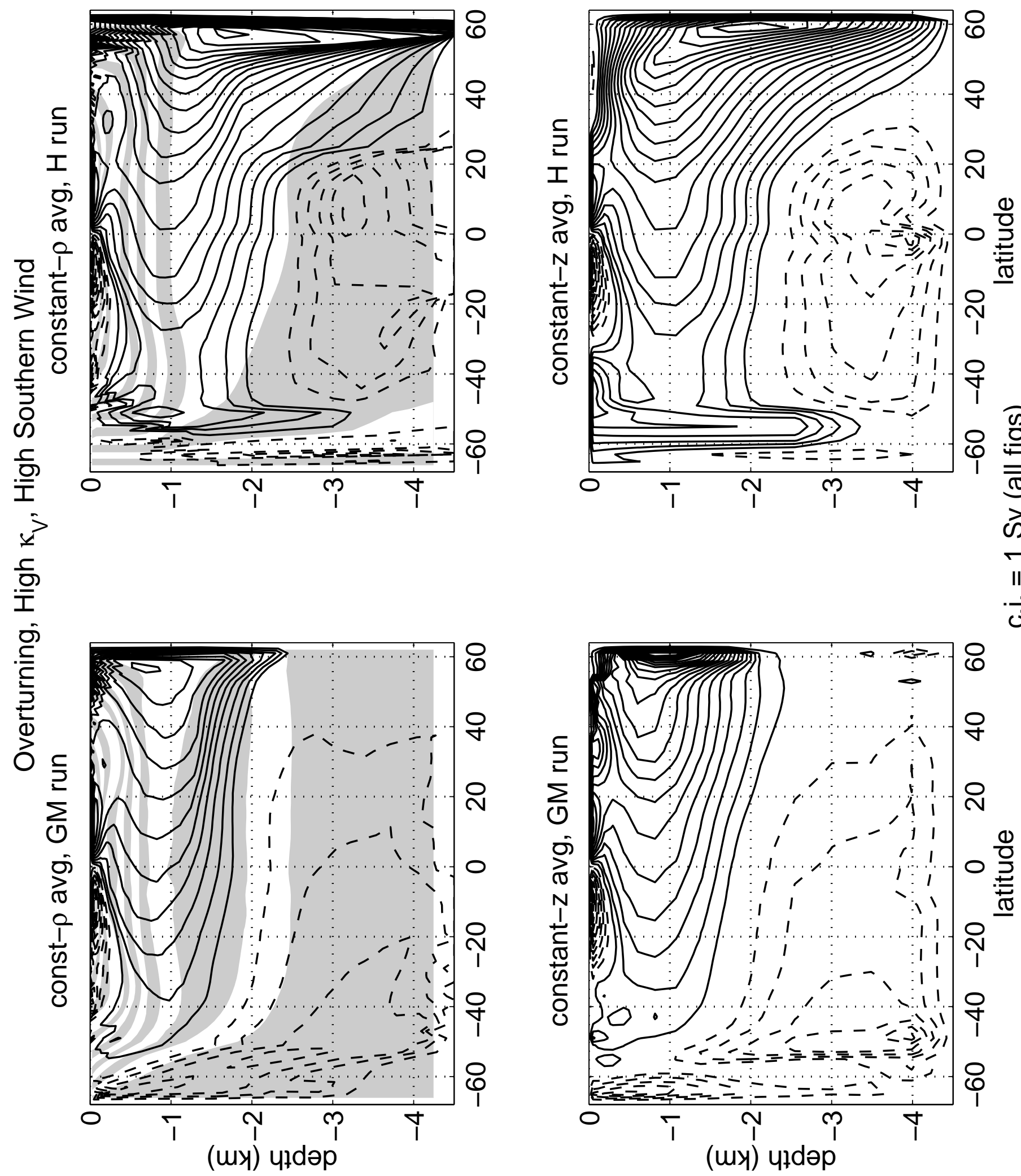

ह
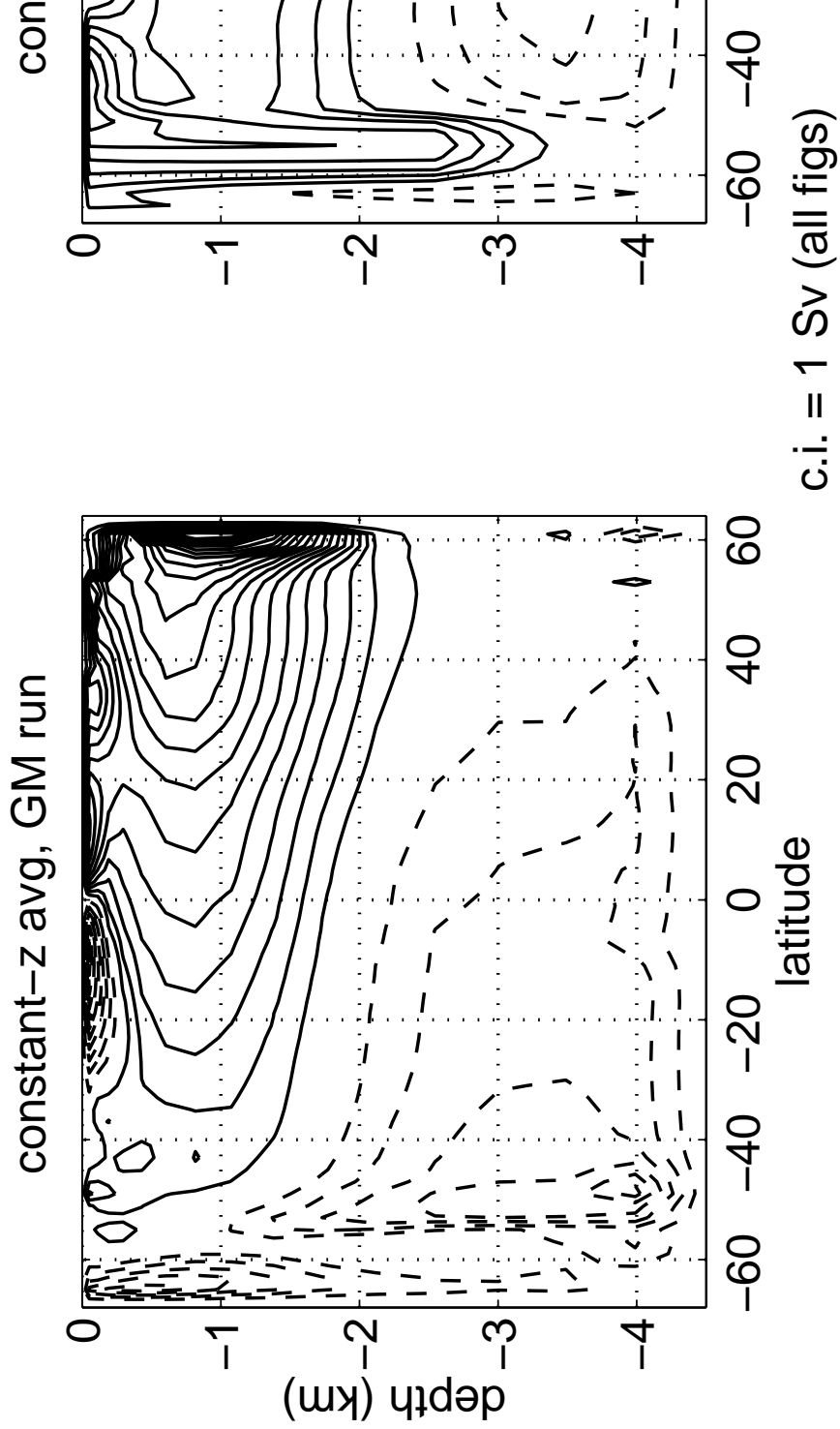


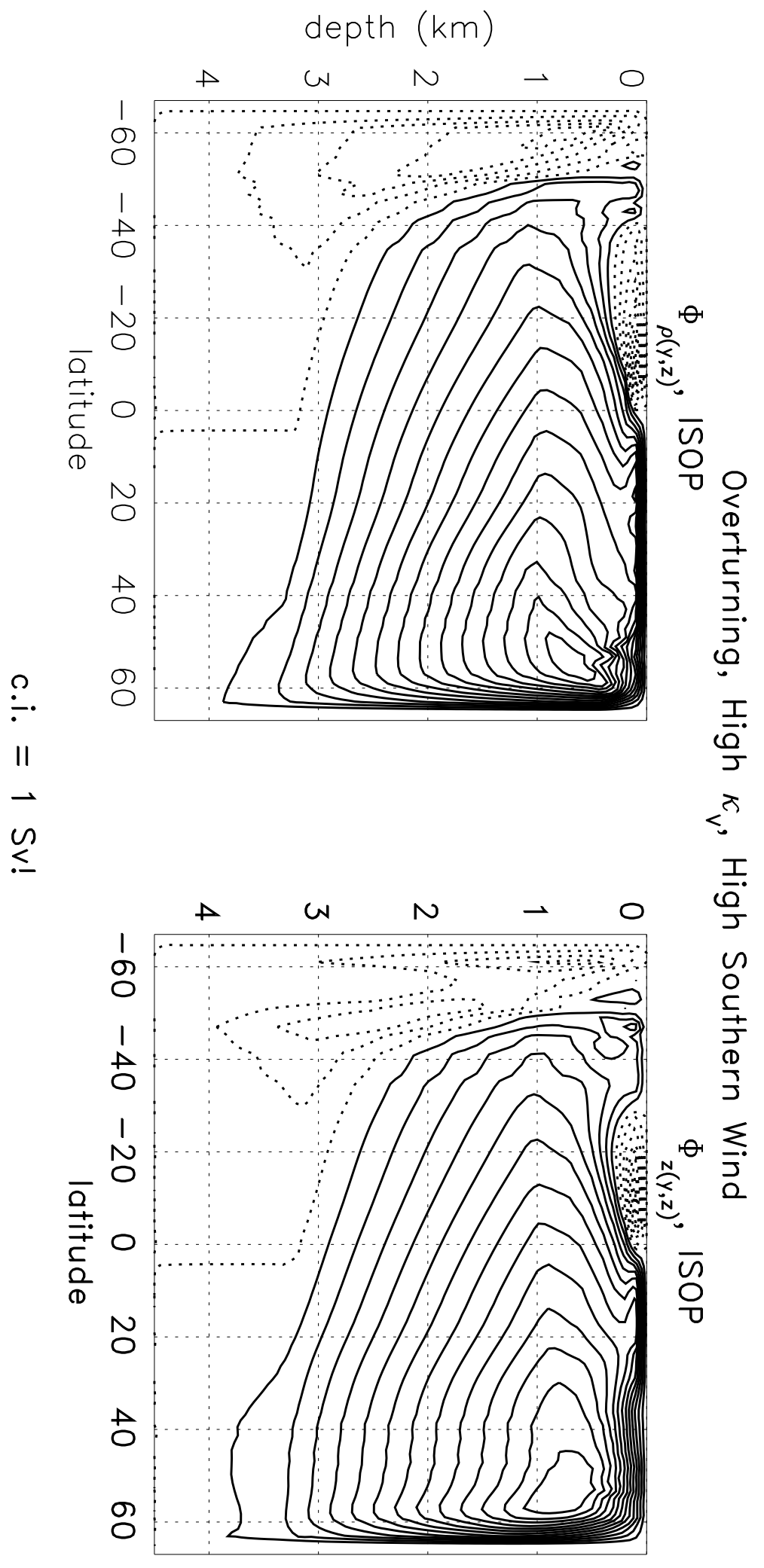



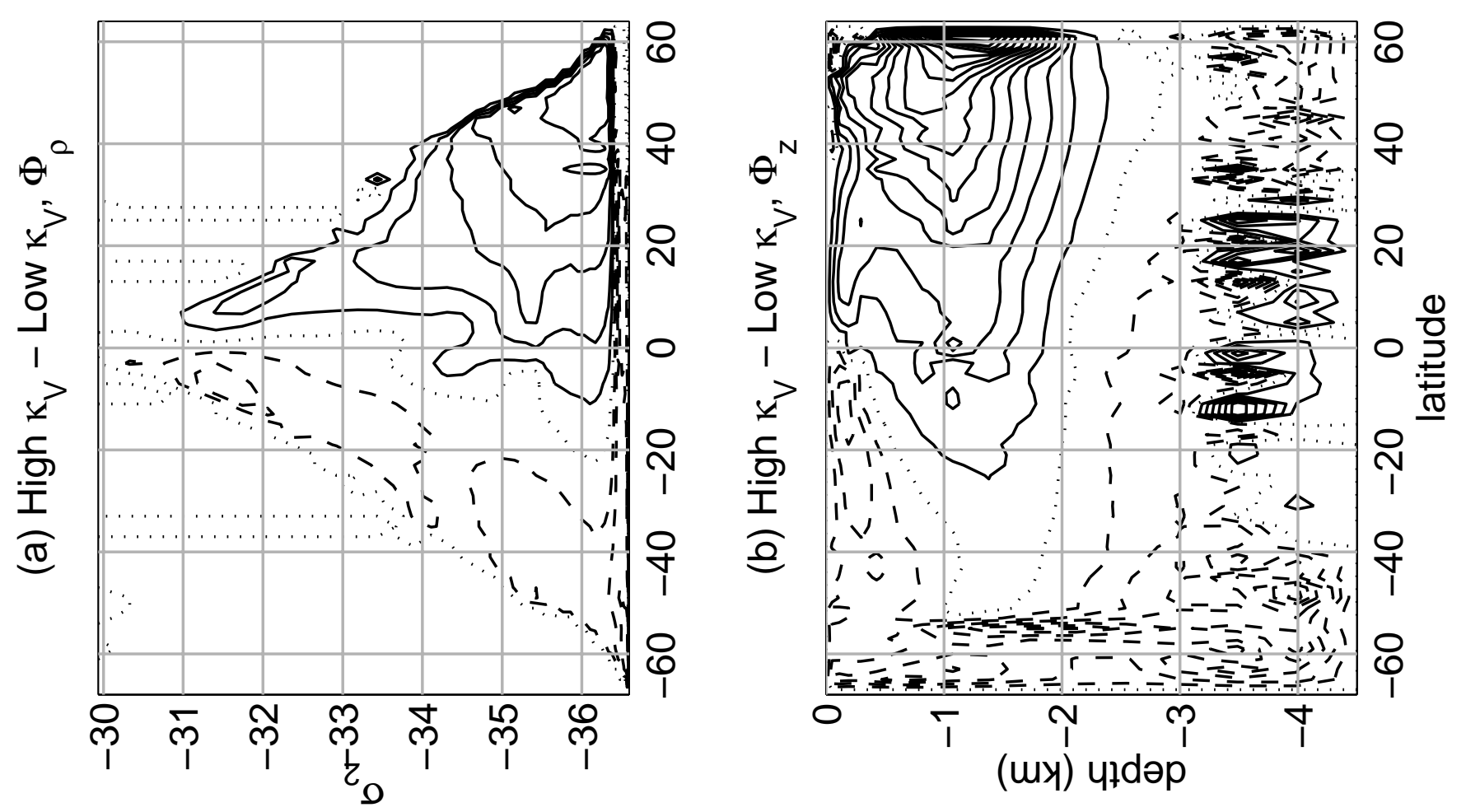


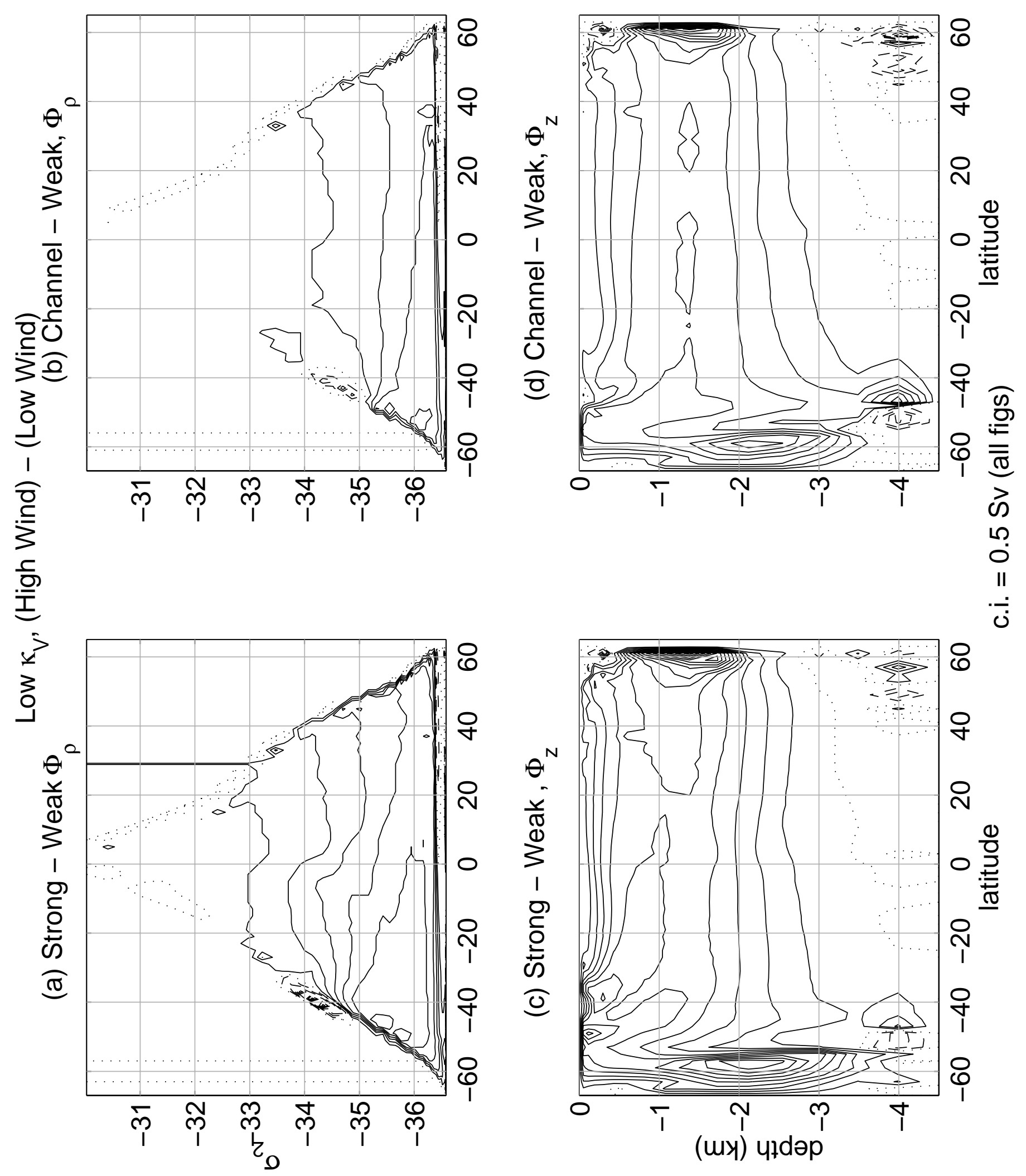



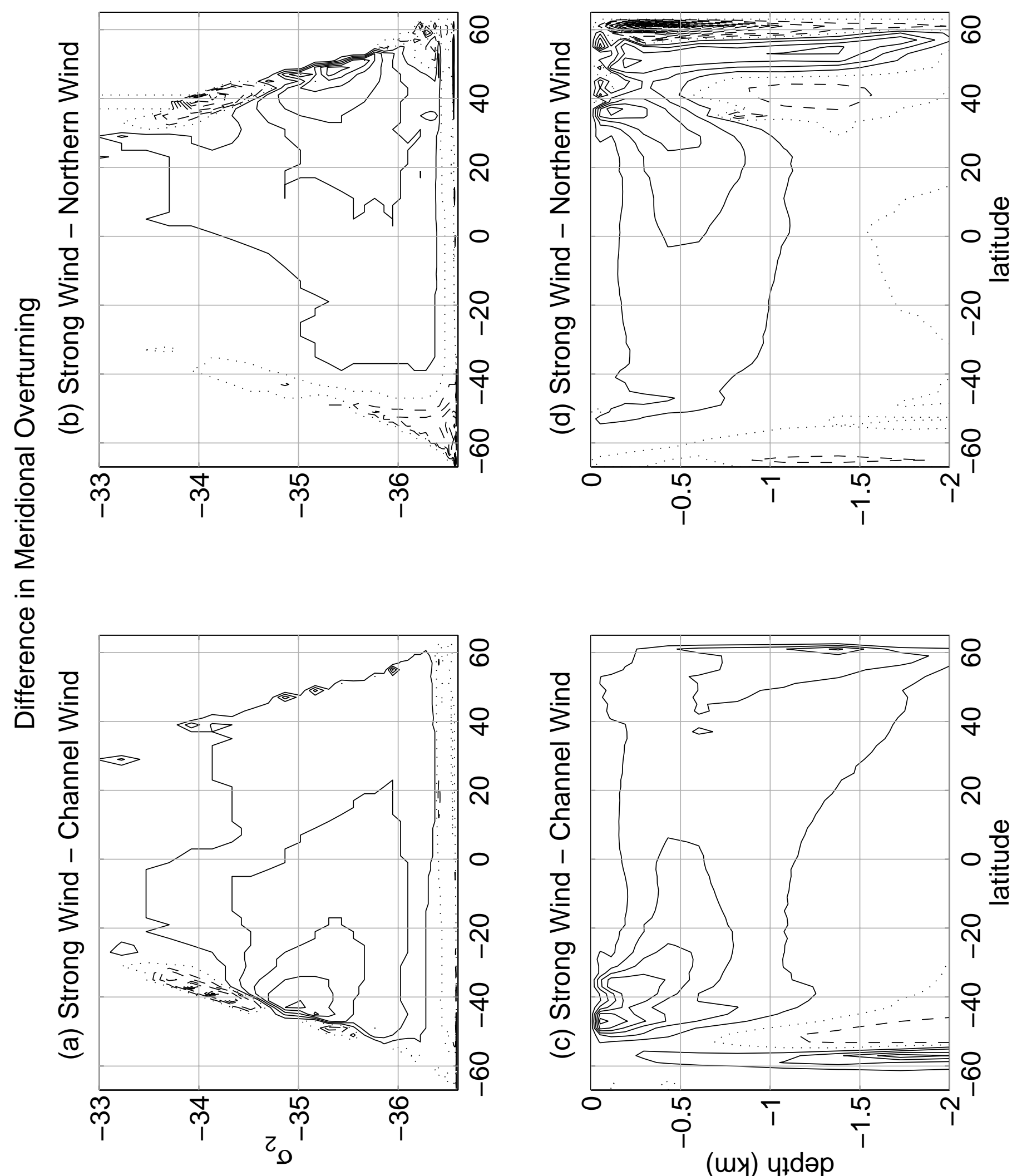

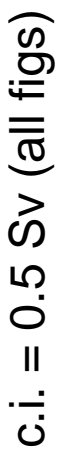

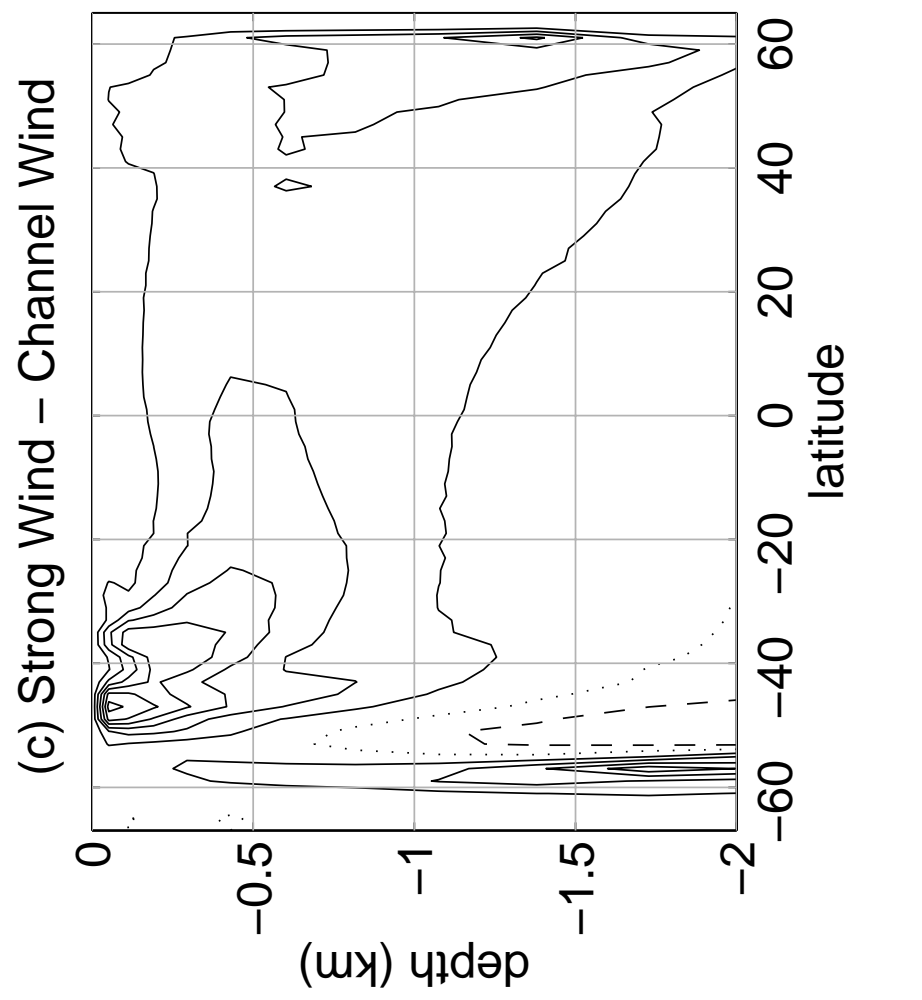



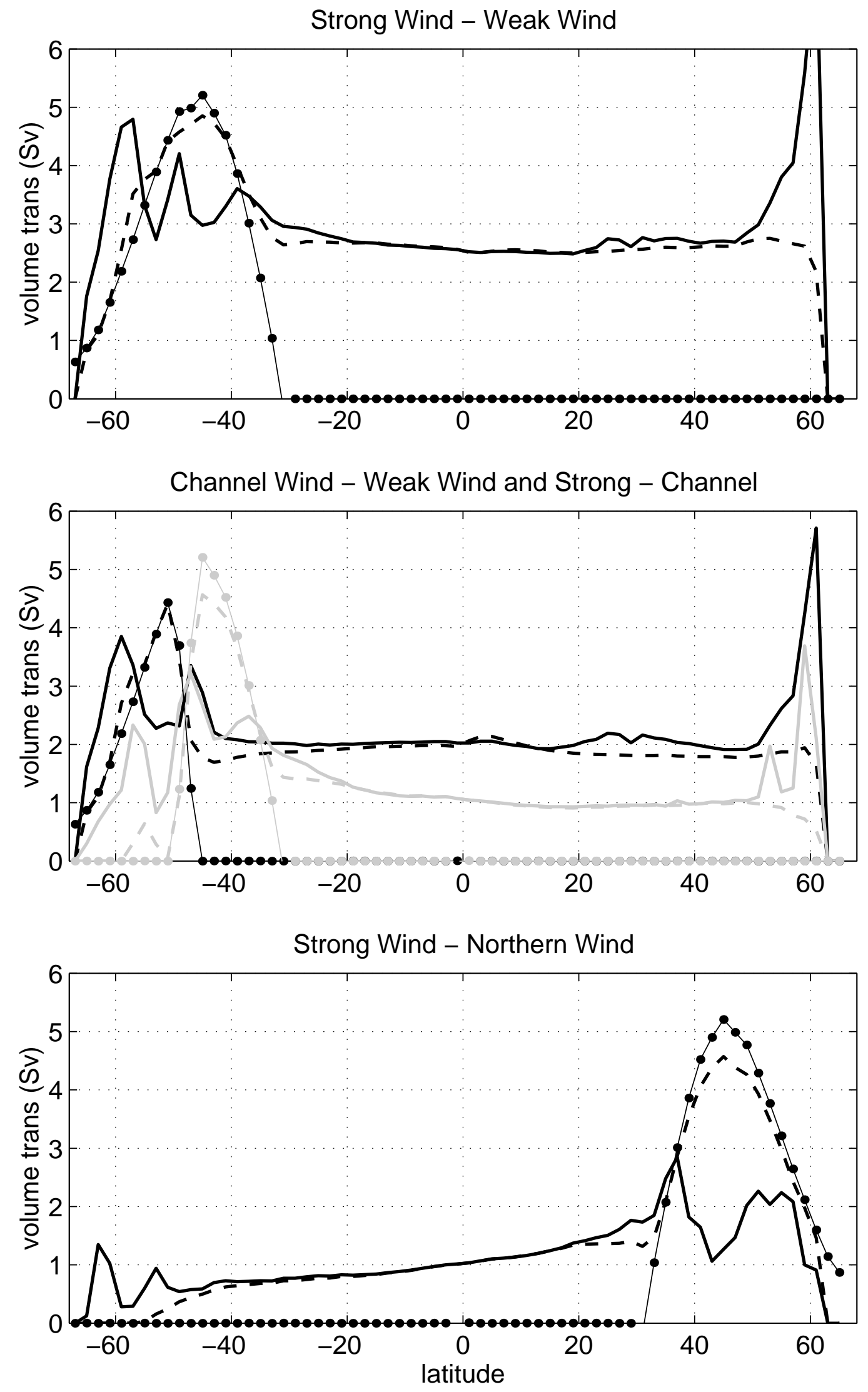
(a) GM

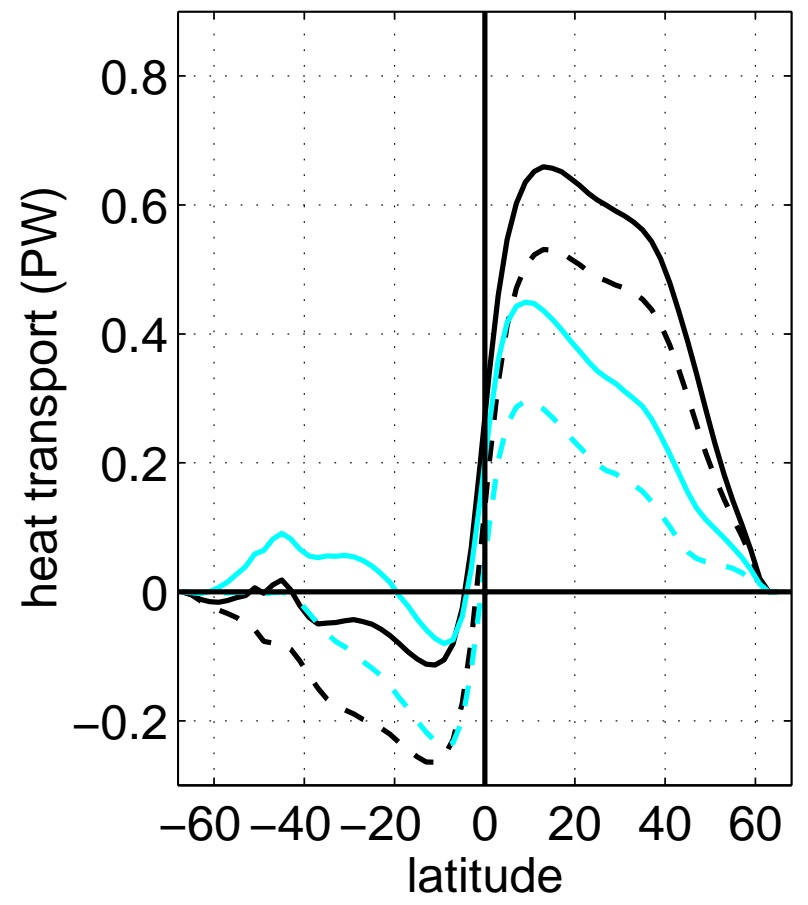

(c) isopycnal model

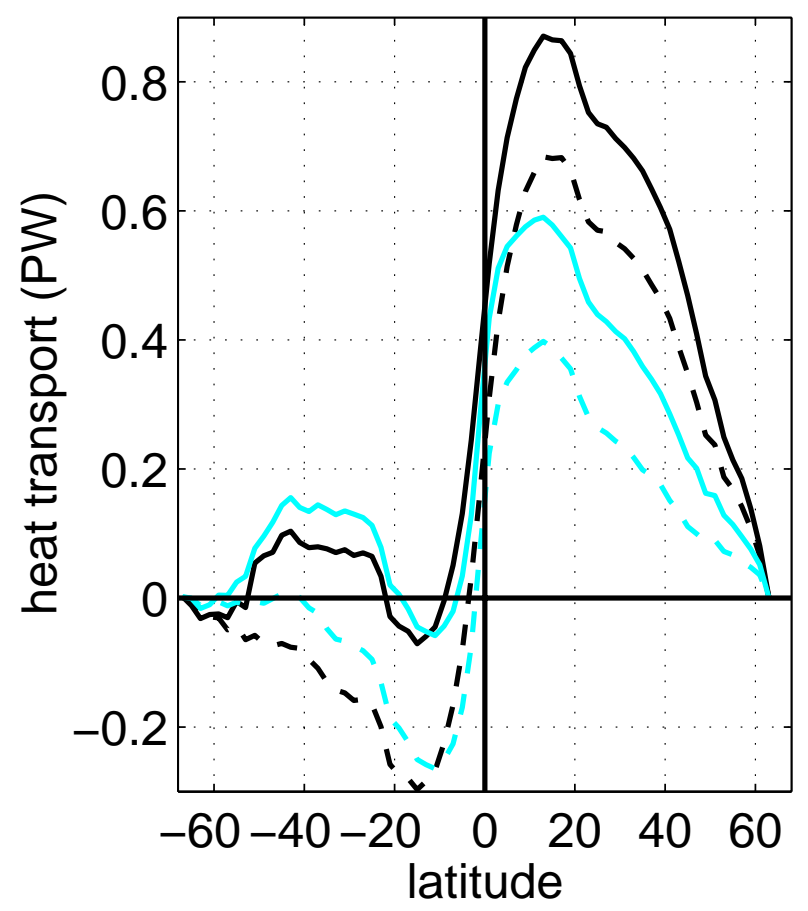

(b) $\mathrm{H}$

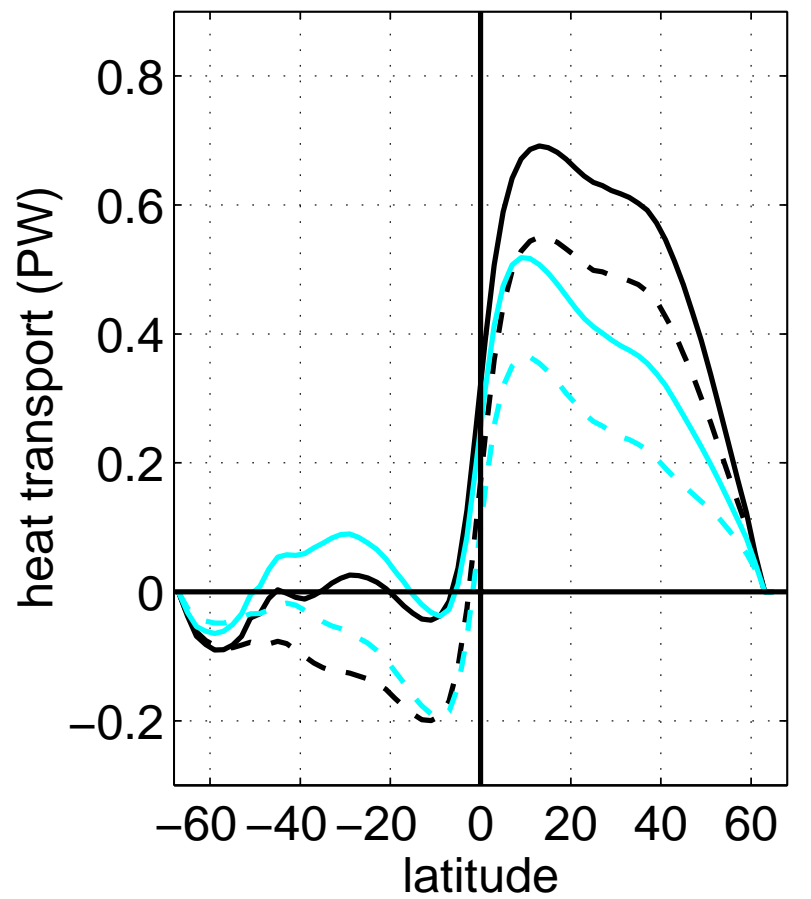




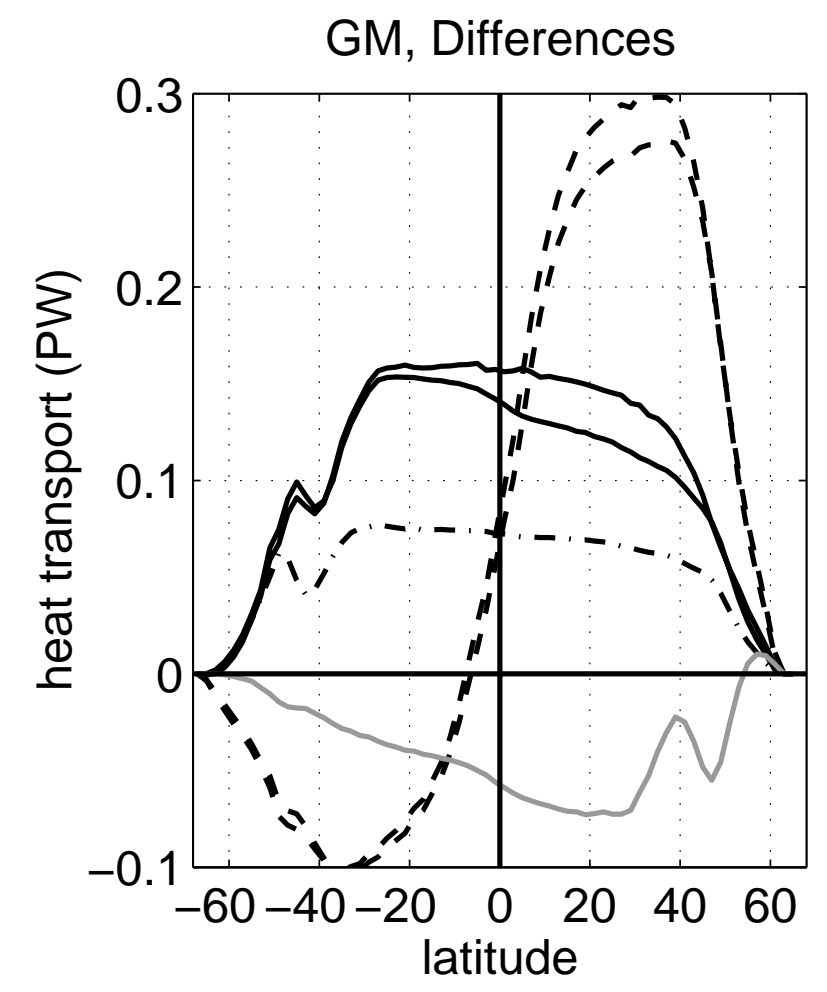


(a) Strong Wind Anom, $\mathbf{u}^{1 / 2}, 25 \mathrm{~m}$

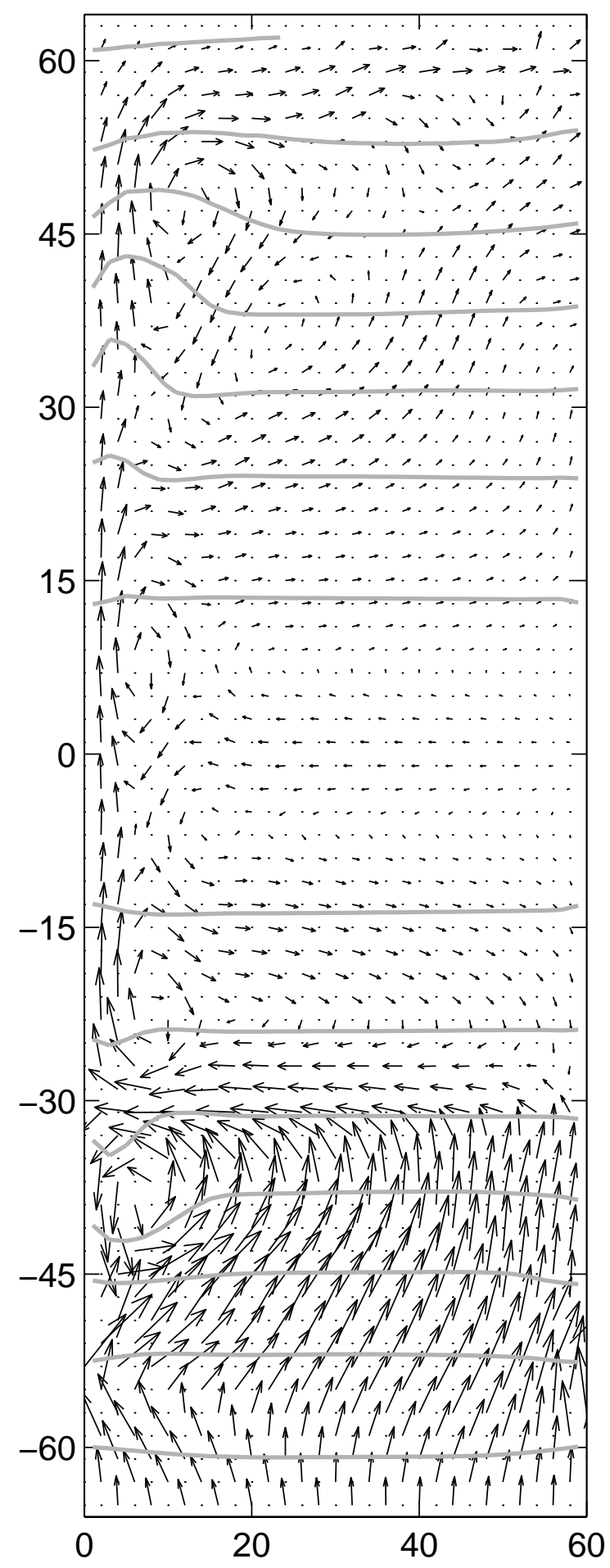

(b) Channel Wind Anom, $\mathbf{u}^{1 / 2}, 25 \mathrm{~m}$

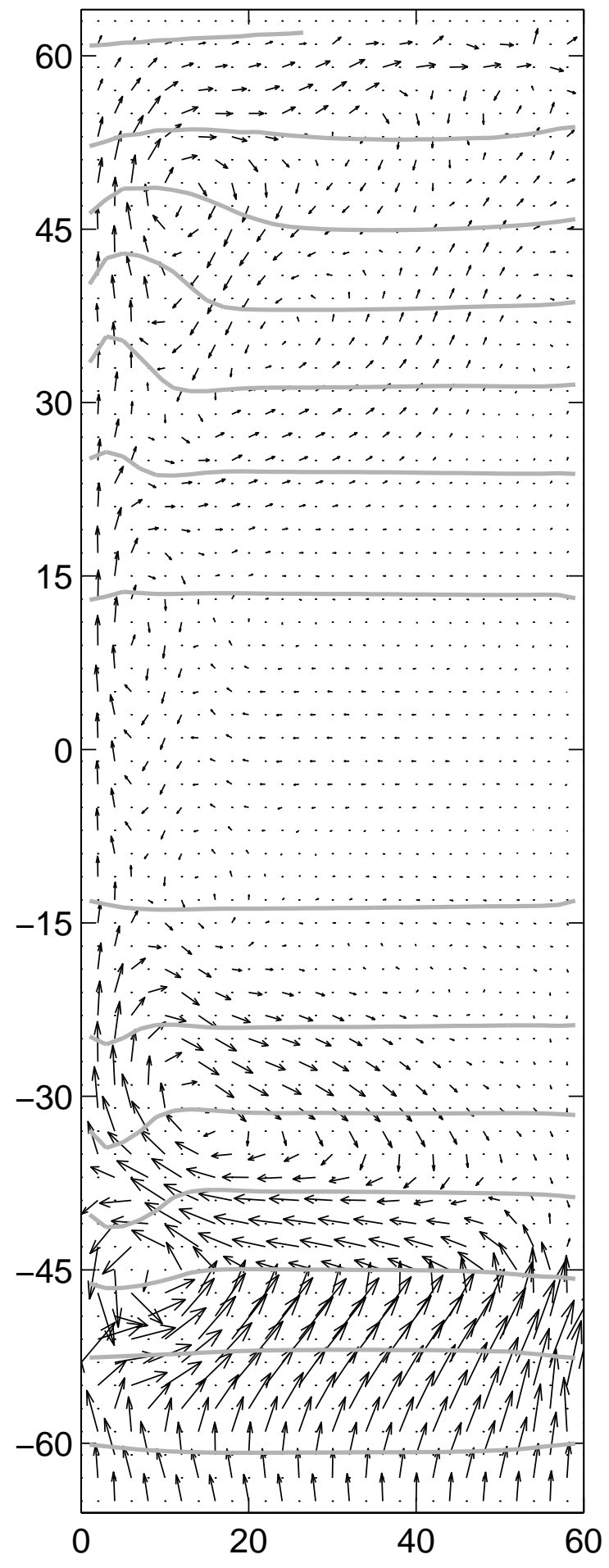

